\title{
TWO-POINT BOUNDARY VALUE PROBLEMS FOR HIGHER-ORDER LINEAR DIFFERENTIAL EQUATIONS WITH STRONG SINGULARITIES
}

\author{
R. P. AGARWAL AND I. KIGURADZE
}

Received 4 April 2004; Revised 11 December 2004; Accepted 14 December 2004

For strongly singular higher-order linear differential equations together with two-point conjugate and right-focal boundary conditions, we provide easily verifiable best possible conditions which guarantee the existence of a unique solution.

Copyright (c) 2006 Hindawi Publishing Corporation. All rights reserved.

\section{Statement of the main results}

1.1. Statement of the problems and the basic notation. Consider the differential equation

$$
u^{(n)}=\sum_{i=1}^{m} p_{i}(t) u^{(i-1)}+q(t)
$$

with the conjugate boundary conditions

$$
\begin{array}{ll}
u^{(i-1)}(a)=0 & (i=1, \ldots, m), \\
u^{(j-1)}(b)=0 & (j=1, \ldots, n-m)
\end{array}
$$

or the right-focal boundary conditions

$$
\begin{array}{ll}
u^{(i-1)}(a)=0 & (i=1, \ldots, m), \\
u^{(j-1)}(b)=0 & (j=m+1, \ldots, n) .
\end{array}
$$

Here $n \geq 2, m$ is the integer part of $n / 2,-\infty<a<b<+\infty, p_{i} \in L_{\mathrm{loc}}(] a, b[)(i=1, \ldots, n)$, $q \in L_{\mathrm{loc}}(] a, b\left[\right.$ ), and by $u^{(i-1)}(a)$ (by $u^{(j-1)}(b)$ ) is understood the right (the left) limit of the function $u^{(i-1)}$ (of the function $u^{(j-1)}$ ) at the point $a$ (at the point $b$ ).

Problems (1.1), (1.2) and (1.1), (1.3) are said to be singular if some or all coefficients of (1.1) are non-integrable on $[a, b]$, having singularities at the ends of this segment. 
2 Linear BVPs with strong singularities

The previous results on the unique solvability of the singular problems (1.1), (1.2) and (1.1), (1.3) deal, respectively, with the cases where

$$
\begin{gathered}
\int_{a}^{b}(t-a)^{n-1}(b-t)^{2 m-1}\left[(-1)^{n-m} p_{1}(t)\right]_{+} d t<+\infty, \\
\int_{a}^{b}(t-a)^{n-i}(b-t)^{2 m-i}\left|p_{i}(t)\right| d t<+\infty \quad(i=2, \ldots, m), \\
\int_{a}^{b}(t-a)^{n-m-1 / 2}(b-t)^{m-1 / 2}|q(t)| d t<+\infty, \\
\int_{a}^{b}(t-a)^{n-1}\left[(-1)^{n-m} p_{1}(t)\right]_{+} d t<+\infty, \\
\int_{a}^{b}(t-a)^{n-i}\left|p_{i}(t)\right| d t<+\infty \quad(i=2, \ldots, m), \\
\int_{a}^{b}(t-a)^{n-m-1 / 2}|q(t)| d t<+\infty
\end{gathered}
$$

(see $[1,2,4,3,5,6,9-18]$, and the references therein).

The aim of the present paper is to investigate problem (1.1), (1.2) (problem (1.1), (1.3)) in the case, where the functions $p_{i}(i=1, \ldots, n)$ and $q$ have strong singularities at the points $a$ and $b$ (at the point $a$ ) and do not satisfy conditions (1.4) (conditions (1.5)).

Throughout the paper we use the following notation.

$[x]_{+}$is the positive part of a number $x$, that is,

$$
[x]_{+}=\frac{x+|x|}{2} .
$$

$\left.\left.L_{\mathrm{loc}}(] a, b[)\left(L_{\mathrm{loc}}(] a, b\right]\right)\right)$ is the space of functions $\left.y:\right] a, b[\rightarrow \mathbb{R}$ which are integrable on $[a+\varepsilon, b-\varepsilon]($ on $[a+\varepsilon, b])$ for arbitrarily small $\varepsilon>0$.

$L_{\alpha, \beta}(] a, b[)\left(L_{\alpha, \beta}^{2}(] a, b[)\right)$ is the space of integrable (square integrable) with the weight $(t-a)^{\alpha}(b-t)^{\beta}$ functions $\left.y:\right] a, b[\rightarrow \mathbb{R}$ with the norm

$$
\|y\|_{L_{\alpha, \beta}}=\int_{a}^{b}(t-a)^{\alpha}(b-t)^{\beta}|y(t)| d t\left(\|y\|_{L_{\alpha, \beta}^{2}}=\left(\int_{a}^{b}(t-a)^{\alpha}(b-t)^{\beta} y^{2}(t) d t\right)^{1 / 2}\right) .
$$

$L([a, b])=L_{0,0}(] a, b[), L^{2}([a, b])=L_{0,0}^{2}(] a, b[)$.

$\left.\left.\widetilde{L}_{\alpha, \beta}^{2}(] a, b[)\left(\widetilde{L}_{\alpha}^{2}(] a, b\right]\right)\right)$ is the space of functions $\left.\left.y \in L_{\mathrm{loc}}(] a, b[)\left(y \in L_{\mathrm{loc}}(] a, b\right]\right)\right)$ such that $\tilde{y} \in L_{\alpha, \beta}^{2}(] a, b[)$, where $\tilde{y}(t)=\int_{c}^{t} y(s) d s, c=(a+b) / 2\left(\tilde{y} \in L_{\alpha, 0}^{2}(] a, b[)\right.$, where $\tilde{y}(t)=$ $\left.\int_{t}^{b} y(s) d s\right)$. 
$\|\cdot\|_{\tilde{L}_{\alpha, \beta}^{2}}$ and $\|\cdot\|_{\widetilde{L}_{\alpha}^{2}}$ denote the norms in $\widetilde{L}_{\alpha, \beta}^{2}(] a, b[)$ and $\left.\left.\widetilde{L}_{\alpha}^{2}(] a, b\right]\right)$, and are defined by the equalities

$$
\begin{aligned}
\|y\|_{\widetilde{L}_{\alpha, \beta}^{2}}= & \max \left\{\left[\int_{a}^{t}(s-a)^{\alpha}\left(\int_{s}^{t} y(\tau) d \tau\right)^{2} d s\right]^{1 / 2}: a \leq t \leq \frac{a+b}{2}\right\} \\
& +\max \left\{\left[\int_{t}^{b}(b-s)^{\beta}\left(\int_{t}^{s} y(\tau) d \tau\right)^{2} d s\right]^{1 / 2}: \frac{a+b}{2} \leq t \leq b\right\}, \\
\|y\|_{\tilde{L}_{\alpha}^{2}}= & \max \left\{\left[\int_{a}^{t}(s-a)^{\alpha}\left(\int_{s}^{t} y(\tau) d \tau\right)^{2} d s\right]^{1 / 2}: a \leq t \leq b\right\} .
\end{aligned}
$$

$\left.\left.\widetilde{C}_{\mathrm{loc}}^{n-1}(] a, b[)\left(\widetilde{C}_{\mathrm{loc}}^{n-1}(] a, b\right]\right)\right)$ is the space of functions $\left.\left.y:\right] a, b[\rightarrow \mathbb{R}(y:] a, b] \rightarrow \mathbb{R}\right)$ which are absolutely continuous together with $y^{\prime}, \ldots, y^{(n-1)}$ on $[a+\varepsilon, b-\varepsilon]($ on $[a+\varepsilon, b])$ for arbitrarily small $\varepsilon>0$.

$\left.\left.\widetilde{C}^{n-1, m}(] a, b[)\left(\widetilde{C}^{n-1, m}(] a, b\right]\right)\right)$ is the space of functions $y \in \widetilde{C}_{\mathrm{loc}}^{n-1}(] a, b[)\left(y \in \widetilde{C}_{\mathrm{loc}}^{n-1}(] a\right.$, b])) such that

$$
\int_{a}^{b}\left|y^{(m)}(s)\right|^{2} d s<+\infty
$$

In what follows, when problem (1.1), (1.2) is discussed, we assume that in the case $n=2 m$ the conditions

$$
p_{i} \in L_{\mathrm{loc}}(] a, b[) \quad(i=1, \ldots, m)
$$

are fulfilled, and in the case $n=2 m+1$ along with (1.10) the condition

$$
\limsup _{t \rightarrow b}\left|(b-t)^{2 m-1} \int_{c}^{t} p_{1}(s) d s\right|<+\infty, \quad c=\frac{a+b}{2}
$$

is also satisfied.

As for problem (1.1), (1.3), it is investigated under the assumptions

$$
\left.\left.p_{i} \in L_{\mathrm{loc}}(] a, b\right]\right) \quad(i=1, \ldots, m)
$$

A solution of problem (1.1), (1.2) (of problem (1.1), (1.3)) is sought in the space $\widetilde{C}^{n-1, m}(] a, b[)$ (in the space $\left.\left.\left.\widetilde{C}^{n-1, m}(] a, b\right]\right)\right)$.

By $\left.h_{i}:\right] a, b[\times] a, b[\rightarrow[0,+\infty[(i=1, \ldots, m)$ we understand the functions defined by the equalities

$$
\begin{gathered}
h_{1}(t, \tau)=\left|\int_{\tau}^{t}(s-a)^{n-2 m}\left[(-1)^{n-m} p_{1}(s)\right]_{+} d s\right|, \\
h_{i}(t, \tau)=\left|\int_{\tau}^{t}(s-a)^{n-2 m} p_{i}(s) d s\right| \quad(i=2, \ldots, m) .
\end{gathered}
$$


1.2. Fredholm type theorems. Along with (1.1), we consider the homogeneous equation

$$
u^{(n)}=\sum_{i=1}^{m} p_{i}(t) u^{(i-1)}
$$

From [10, Corollary 1.1] it follows that if

$$
\begin{aligned}
& p_{i} \in L_{n-m, m}(] a, b[) \quad(i=1, \ldots, m), \\
&\left(p_{i} \in L_{n-m, 0}(] a, b[) \quad(i=1, \ldots, m)\right)
\end{aligned}
$$

and the homogeneous problem $\left(1.1_{0}\right),(1.2)$ (problem $\left.\left(1.1_{0}\right),(1.3)\right)$ has only a trivial solution in the space $\widetilde{C}_{\mathrm{loc}}^{n-1}(] a, b[)$ (in the space $\left.\left.\widetilde{C}_{\mathrm{loc}}^{n-1}(] a, b\right]\right)$ ), then for every $q \in L_{n-m, m}(] a, b[)$ $\left(q \in L_{n-m, 0}(] a, b[)\right)$ problem (1.1), (1.2) (problem (1.1), (1.3)) is uniquely solvable in the space $\widetilde{C}_{\mathrm{loc}}^{n-1}(] a, b[)$ (in the space $\left.\left.\left.\widetilde{C}_{\mathrm{loc}}^{n-1}(] a, b\right]\right)\right)$.

In the case where condition (1.14) is violated, the question on the presence of the Fredholm property for problem (1.1), (1.2) (for problem (1.1), (1.3)) in some subspace of the space $\widetilde{C}_{\mathrm{loc}}^{n-1}(] a, b[)$ (of the space $\left.\left.\left.\widetilde{C}_{\mathrm{loc}}^{n-1}(] a, b\right]\right)\right)$ remained so far open. This question is answered in Theorem 1.3 (Theorem 1.5) formulated below which contains optimal in a certain sense conditions guaranteeing the presence of the Fredholm property for problem (1.1), (1.2) (for problem $(1.1),(1.3))$ in the space $\widetilde{C}^{n-1, m}(] a, b[)$ (in the space $\left.\left.\left.\widetilde{C}^{n-1, m}(] a, b\right]\right)\right)$.

Definition 1.1. We say that problem (1.1), (1.2) (problem (1.1), (1.3)) has the Fredholm property in the space $\widetilde{C}^{n-1, m}(] a, b\left[\right.$ ) (in the space $\left.\left.\left.\widetilde{C}^{n-1, m}(] a, b\right]\right)\right)$ if the unique solvability of the corresponding homogeneous problem $\left(1.1_{0}\right),(1.2)$ (problem $\left(1.1_{0}\right),(1.3)$ ) in this space implies the unique solvability of problem (1.1), (1.2) (problem (1.1), (1.3)) in the space $\widetilde{C}^{n-1, m}(] a, b\left[\right.$ ) (in the space $\left.\left.\widetilde{C}^{n-1, m}(] a, b\right]\right)$ ) for every $q \in \widetilde{L}_{2 n-2 m-2,2 m-2}^{2}(] a, b[$ ) (for every $\left.\left.\left.q \in \widetilde{L}_{2 n-2 m-2}^{2}(] a, b\right]\right)\right)$ and for its solution the following estimate

$$
\left\|u^{(m)}\right\|_{L^{2}} \leq r\|q\|_{\tilde{L}_{2 n-2 m-2,2 m-2}^{2}} \quad\left(\left\|u^{(m)}\right\|_{L^{2}} \leq r\|q\|_{\tilde{L}_{2 n-2 m-2}^{2}}\right)
$$

is valid, where $r$ is a positive constant independent of $q$.

Remark 1.2. If

$$
q \in L_{2 n-2 m, 2 m}^{2}(] a, b[) \quad\left(q \in L_{2 n-2 m, 0}^{2}(] a, b[)\right)
$$

or

$$
q \in L_{n-m-1 / 2, m-1 / 2}(] a, b[) \quad\left(q \in L_{n-m-1 / 2,0}(] a, b[)\right),
$$

then

$$
\left.\left.q \in \widetilde{L}_{2 n-2 m-2,2 m-2}^{2}(] a, b[) \quad\left(q \in \widetilde{L}_{2 n-2 m-2}^{2}(] a, b\right]\right)\right)
$$


and from estimate (1.15) there respectively follow the estimates

$$
\begin{array}{lr}
\left\|u^{(m)}\right\|_{L^{2}} \leq r_{0}\|q\|_{L_{2 n+2 m, 2 m}^{2}} & \left(\left\|u^{(m)}\right\|_{L^{2}} \leq r_{0}\|q\|_{L_{2 n-2 m, 0}^{2}}\right), \\
\left\|u^{(m)}\right\|_{L^{2}} \leq r_{0}\|q\|_{L_{n-m-1 / 2, m-1 / 2}} & \left(\left\|u^{(m)}\right\|_{L^{2}} \leq r_{0}\|q\|_{L_{n-m-1 / 2,0}}\right),
\end{array}
$$

where $r_{0}$ is a positive constant independent of $q$.

Theorem 1.3. Let there exist $\left.a_{0} \in\right] a, b\left[, b_{0} \in\right] a_{0}, b$ [ and nonnegative numbers $\ell_{1 i}, \ell_{2 i}(i=$ $1, \ldots, m)$ such that

$$
\begin{gathered}
(t-a)^{2 m-i} h_{i}(t, \tau) \leq \ell_{1 i} \quad \text { for } a<t \leq \tau \leq a_{0}, \\
(b-t)^{2 m-i} h_{i}(t, \tau) \leq \ell_{2 i} \quad \text { for } b_{0} \leq \tau \leq t<b(i=1, \ldots, m), \\
\sum_{i=1}^{m} \frac{(2 m-i) 2^{n-i+1}}{(2 m-2 i+1) ! !} \ell_{1 i}<(2 n-2 m-1) ! ! \\
\sum_{i=1}^{m} \frac{(2 m-i) 2^{n-i+1}}{(2 m-2 i+1) ! !} \ell_{2 i}<(2 n-2 m-1) ! !
\end{gathered}
$$

where $(2 n-2 i-1) ! !=1.3 \cdots(2 n-2 i-1)$. Then problem (1.1), (1.2) has the Fredholm property in the space $\tilde{C}^{n-1, m}(] a, b[)$.

Corollary 1.4. Let there exist nonnegative numbers $\lambda_{1 i}, \lambda_{2 i}(i=1, \ldots, m)$ and functions $p_{0 i} \in L_{n-i, 2 m-i}(] a, b[)(i=1, \ldots, m)$ such that the inequalities

$$
\begin{gathered}
(-1)^{n-m} p_{1}(t) \leq \frac{\lambda_{11}}{(t-a)^{n}}+\frac{\lambda_{21}}{(t-a)^{n-2 m}(b-t)^{2 m}}+p_{01}(t), \\
\left|p_{i}(t)\right| \leq \frac{\lambda_{1 i}}{(t-a)^{n-i+1}}+\frac{\lambda_{2 i}}{(t-a)^{n-2 m}(b-t)^{2 m-i+1}}+p_{0 i}(t) \quad(i=2, \ldots, m)
\end{gathered}
$$

hold almost everywhere on $] a, b[$, and

$$
\begin{aligned}
& \sum_{i=1}^{m} \frac{2^{n-i+1}}{(2 m-2 i+1) ! !} \lambda_{1 i}<(2 n-2 m-1) ! ! \\
& \sum_{i=1}^{m} \frac{2^{n-i+1}}{(2 m-2 i+1) ! !} \lambda_{2 i}<(2 n-2 m-1) ! !
\end{aligned}
$$

Then problem (1.1), (1.2) has the Fredholm property in the space $\widetilde{C}^{n-1, m}(] a, b[)$.

Theorem 1.5. Let there exist $\left.a_{0} \in\right] a, b\left[\right.$ and nonnegative numbers $\ell_{i}(i=1, \ldots, m)$ such that

$$
\begin{gathered}
(t-a)^{2 m-i} h_{i}(t, \tau) \leq \ell_{i} \quad \text { for } a<t \leq \tau \leq a_{0}(i=1, \ldots, m), \\
\sum_{i=1}^{m} \frac{(2 m-i) 2^{n-i+1}}{(2 m-2 i+1) ! !} \ell_{i}<(2 n-2 m-1) ! !
\end{gathered}
$$

Then problem (1.1), (1.3) has the Fredholm property in the space $\left.\left.\widetilde{C}^{n-1, m}(] a, b\right]\right)$. 
Corollary 1.6. Let there exist nonnegative numbers $\lambda_{i}(i=1, \ldots, m)$ and functions $p_{0 i} \in$ $L_{n-i, 0}(] a, b[)(i=1, \ldots, m)$ such that the inequalities

$$
\begin{gathered}
(-1)^{n-m} p_{1}(t) \leq \frac{\lambda_{1}}{(t-a)^{n}}+p_{01}(t), \\
\left|p_{i}(t)\right| \leq \frac{\lambda_{i}}{(t-a)^{n-i+1}}+p_{0 i}(t) \quad(i=2, \ldots, m)
\end{gathered}
$$

hold almost everywhere on $] a, b[$, and

$$
\sum_{i=1}^{m} \frac{2^{n-i+1}}{(2 m-2 i+1) ! !} \lambda_{i}<(2 n-2 m-1) ! !
$$

Then problem (1.1), (1.3) has the Fredholm property in the space $\left.\left.\widetilde{C}^{n-1, m}(] a, b\right]\right)$.

In connection with the above-mentioned Corollary 1.1 from [10], there naturally arises the problem of finding the conditions under which the unique solvability of problem (1.1), (1.2) (of problem (1.1), (1.3)) in the space $\widetilde{C}^{n-1, m}(] a, b[$ ) (in the space $\left.\left.\left.\widetilde{C}^{n-1, m}(] a, b\right]\right)\right)$ guarantees the unique solvability of that problem in the space $\widetilde{C}_{\text {loc }}^{n-1}(] a, b[)$ (in the space $\left.\left.\widetilde{C}_{\text {loc }}^{n-1}(] a, b\right]\right)$ ).

The following theorem is valid.

THEOREM 1.7. If

$$
\begin{gathered}
p_{i} \in L_{n-i, 2 m-i}(] a, b[) \quad(i=1, \ldots, m), \\
\left(p_{i} \in L_{n-i, 0}(] a, b[) \quad(i=1, \ldots, m)\right),
\end{gathered}
$$

and problem (1.1), (1.2) (problem (1.1), (1.3)) is uniquely solvable in the space $\widetilde{C}^{n-1, m}(] a$, $b\left[\right.$ ) (in the space $\left.\left.\widetilde{C}^{n-1, m}(] a, b\right]\right)$ ), then this problem is uniquely solvable in the space $\widetilde{C}_{\mathrm{loc}}^{n-1}(] a$, $b\left[\right.$ ) (in the space $\left.\left.\widetilde{C}_{\mathrm{loc}}^{n-1}(] a, b\right]\right)$ ) as well.

If condition (1.28) is violated, then, as it is clear from the example below, problem (1.1), (1.2) (problem (1.1), (1.3)) may be uniquely solvable in the space $\widetilde{C}^{n-1, m}(] a, b[$ ) (in the space $\left.\left.\left.\widetilde{C}^{n-1, m}(] a, b\right]\right)\right)$ and this problem may have an infinite set of solutions in the space $\widetilde{C}_{\mathrm{loc}}^{n-1}(] a, b[)$ (in the space $\left.\left.\left.\widetilde{C}_{\mathrm{loc}}^{n-1}(] a, b\right]\right)\right)$.

Example 1.8. Suppose

$$
g_{n}(x)=x(x-1) \cdots(x-n+1) .
$$

Then

$$
\begin{gathered}
(-1)^{n-m} g_{n}\left(m-\frac{1}{2}\right)=2^{-n}(2 m-1) ! !(2 n-2 m-1) ! !, \\
g_{n}^{\prime}\left(m-\frac{1}{2}\right)=0 \quad \text { for } n=2 m, \quad g_{n}^{\prime}\left(m-\frac{1}{2}\right) g_{n}\left(m-\frac{1}{2}\right)<0 \quad \text { for } n=2 m+1, \\
(-1)^{n-m} g_{n}\left(k-\frac{1}{2}\right)>(-1)^{n-m} g_{n}\left(m-\frac{1}{2}\right) \quad \text { for } k \in\{0, \ldots, n\} \text { and } m-k \text { is even. }
\end{gathered}
$$


If

$$
p_{1}(t)=\frac{\lambda}{(t-a)^{n}}, \quad p_{i}(t)=0 \quad(i=2, \ldots, n)
$$

and $q(t)=\left(g_{n}(\nu)-\lambda\right) t^{\nu-n}$, where $\lambda \neq 0, v>0$, then (1.1) and (1.1 $)$ have the forms

$$
\begin{gathered}
u^{(n)}=\frac{\lambda}{(t-a)^{n}} u+\left(g_{n}(\nu)-\lambda\right)(t-a)^{\nu-n} \\
u^{(n)}=\frac{\lambda}{(t-a)^{n}} u
\end{gathered}
$$

First we consider the case where

$$
\lambda=g_{n}\left(m-\frac{1}{2}\right)
$$

Then from (1.31) and (1.32) it easily follows that the characteristic equation

$$
g_{n}(x)=\lambda
$$

has only real roots $x_{i}(i=1, \ldots, n)$ such that

$$
\begin{gathered}
x_{1}=x_{2}=\frac{1}{2} \quad \text { for } n=2, \\
x_{1}>\cdots>x_{m-1}>m-\frac{1}{2}=x_{m}=x_{m+1}>\cdots>x_{2 m} \quad \text { for } n=2 m, \\
x_{1}>\cdots>x_{m}>m-\frac{1}{2}>x_{m+1}>\cdots>x_{2 m+1} \quad \text { for } n=2 m+1 .
\end{gathered}
$$

Hence it is evident that for $n=2\left(1.34_{0}\right)$ does not have a solution belonging to the space $\widetilde{C}^{1,1}(] a, b[)$, and for $n>2$ solutions of that equation from the space $\tilde{C}^{n-1, m}(] a, b[)$ constitute an $(n-m-1)$-dimensional subspace with the basis

$$
(t-a)^{x_{1}}, \ldots,(t-a)^{x_{n-m-1}}
$$

Thus problem $\left(1.34_{0}\right),(1.2)$ (problem $\left.\left(1.34_{0}\right),(1.3)\right)$ has only a trivial solution in the space $\widetilde{C}^{n-1, m}(] a, b[)$. We show that nevertheless problem (1.34), (1.2) (problem (1.34), (1.3)) does not have a solution in the space $\widetilde{C}^{n-1, m}(] a, b[)$. Indeed, if $n=2$, then (1.34) has the unique solution $u(t)=(t-a)^{v}$ in the space $\widetilde{C}^{1,1}(] a, b[)$, and this solution does not satisfy conditions (1.2). If $n>2$, then an arbitrary solution of (1.34) from $\widetilde{C}^{n-1, m}(] a, b[)$ has the form

$$
u(t)=\sum_{i=1}^{n-m-1} c_{i}(t-a)^{x_{i}}+(t-a)^{\nu}
$$


and this solution satisfies the boundary conditions (1.2) (the boundary conditions (1.3)) if and only if $c_{1}, \ldots, c_{n-m-1}$ are solutions of the system of linear algebraic equations

$$
\begin{gathered}
\sum_{i=1}^{n-m-1} g_{k}\left(x_{i}\right)(b-a)^{x_{i}} c_{i}=-g_{k}(\nu)(b-a)^{\nu} \quad(k=0, \ldots, n-m-1) \\
\left(\sum_{i=1}^{n-m-1} g_{k}\left(x_{i}\right)(b-a)^{x_{i}} c_{i}=-g_{k}(\nu)(b-a)^{\nu} \quad(k=m, \ldots, n-1)\right),
\end{gathered}
$$

where $g_{0}(x) \equiv 1, g_{k}(x)=x(x-1) \cdots(x-k+1)$ for $x \geq 1$. However, this system does not have a solution for large $\nu$.

Note that in the case under consideration the functions $p_{i}(i=1, \ldots, m)$ in view of conditions (1.30) and (1.32) satisfy inequalities (1.22) (inequalities (1.26)), where $\lambda_{11}=|\lambda|$, $\lambda_{1 i}=\lambda_{21}=\lambda_{2 i}=0(i=2, \ldots, m)\left(\lambda_{1}=|\lambda|, \lambda_{i}=0(i=2, \ldots, m)\right), p_{0 i}(t) \equiv 0(i=1, \ldots, m)$, and

$$
\begin{gathered}
\sum_{i=0}^{m} \frac{2^{n-i+1}}{(2 m-2 i+1) ! !} \lambda_{1 i}=(2 n-2 m-1) ! ! \\
\left(\sum_{i=0}^{m} \frac{2^{n-i+1}}{(2 m-2 i+1) ! !} \lambda_{i}=(2 n-2 m-1) ! !\right) .
\end{gathered}
$$

Therefore we showed that in Theorems 1.3, 1.5 and their corollaries none of strict inequalities (1.21), (1.23), (1.25), and (1.27) can be replaced by nonstrict ones, and in this sense the above-given conditions on the presence of the Fredholm property for problems (1.1), (1.2) and (1.1), (1.3) are the best possible.

Now we consider the case, where

$$
0<(-1)^{n-m} \lambda<(-1)^{n-m} g_{n}\left(m-\frac{1}{2}\right) .
$$

Then, in view of (1.30) and (1.33), the functions $p_{i}(i=1, \ldots, m)$ satisfy all the conditions of Corollaries 1.4 and 1.6, but condition (1.28) in Theorem 1.7 is violated. On the other hand, according to conditions (1.31) and (1.32), the characteristic equation (1.36) has simple real roots $x_{1}, \ldots, x_{n}$ such that

$$
x_{1}>\cdots>x_{n-m}>m-\frac{1}{2}>x_{n-m+1}>\cdots>x_{n},
$$

at that

$$
x_{n-m+1}>m-1 \text {. }
$$

So, the set of solutions of $\left(1.34_{0}\right)$ from $\widetilde{C}^{n-1, m}(] a, b[)$ constitutes an $(n-m)$-dimensional subspace with the basis

$$
(t-a)^{x_{1}}, \ldots,(t-a)^{x_{n-m}},
$$


and consequently, both problem $\left(1.34_{0}\right),(1.2)$ and problem $\left(1.34_{0}\right),(1.3)$ in the mentioned space have only trivial solutions. Hence in view of Corollaries 1.4 and 1.6 the unique solvability of problems (1.34), (1.2) and (1.34), (1.3) follows in $\widetilde{C}^{n-1, m}(] a, b[)$. Let us show that these problems in $\left.\left.\widetilde{C}_{\mathrm{loc}}^{n-1}(] a, b\right]\right)$ have infinite sets of solutions. Indeed, for any $c_{i} \in \mathbb{R}(i=1, \ldots, n-m+1)$, the function

$$
u(t)=\sum_{i=1}^{n-m+1} c_{i}(t-a)^{x_{i}}+(t-a)^{v}
$$

is a solution of (1.34) from $\left.\left.\widetilde{C}_{\mathrm{loc}}^{n-1}(] a, b\right]\right)$, satisfying the conditions

$$
u^{(i-1)}(a)=0 \quad(i=1, \ldots, m) .
$$

This function satisfies the boundary conditions (1.2) (the boundary conditions (1.3)) if and only if $c_{1}, \ldots, c_{n-m}$ are solutions of the system of algebraic equations

$$
\begin{aligned}
& \sum_{i=1}^{n-m} g_{k}\left(x_{i}\right)(b-a)^{x_{i}} c_{i}= \\
& \quad-g_{k}\left(x_{n-m+1}\right)(b-a)^{x_{n-m+1}} c_{n-m+1}-g_{k}(\nu)(b-a)^{\nu}(k=0, \ldots, n-m-1) \\
& \left(\sum_{i=1}^{n-m} g_{k}\left(x_{i}\right)(b-a)^{x_{i}} c_{i}=\right. \\
& \left.\quad-g_{k}\left(x_{n-m+1}\right)(b-a)^{x_{n-m+1}} c_{n-m+1}-g_{k}(\nu)(b-a)^{\nu}(k=n-m, \ldots, m)\right)
\end{aligned}
$$

for any $c_{n-m+1} \in \mathbb{R}$. However, this system has a unique solution for an arbitrarily fixed $c_{n-m+1}$. Thus problem (1.34), (1.2) (problem (1.34), (1.3)) has a one-parameter family of solutions in the space $\left.\left.\widetilde{C}_{\mathrm{loc}}^{n-1}(] a, b\right]\right)$.

\subsection{Existence and uniqueness theorems.}

THeorem 1.9. Let there exist $\left.t_{0} \in\right] a, b\left[\right.$ and nonnegative numbers $\ell_{1 i}, \ell_{2 i}(i=1, \ldots, m)$ such that along with (1.21) the conditions

$$
\begin{array}{ll}
(t-a)^{2 m-i} h_{i}(t, \tau) \leq \ell_{1 i} & \text { for } a<t \leq \tau \leq t_{0}, \\
(b-t)^{2 m-i} h_{i}(t, \tau) \leq \ell_{2 i} & \text { for } t_{0} \leq \tau \leq t<b
\end{array}
$$

hold. Then for every $q \in \tilde{L}_{2 n-2 m-2,2 m-2}^{2}(] a, b[)$ problem (1.1), (1.2) is uniquely solvable in the space $\widetilde{C}^{n-1, m}(] a, b[)$.

Corollary 1.10. Let there exist $\left.t_{0} \in\right] a, b\left[\right.$ and nonnegative numbers $\lambda_{1 i}, \lambda_{2 i}(i=1, \ldots, m)$ such that conditions (1.23) are fulfilled, the inequalities

$$
(-1)^{n-m}(t-a)^{n} p_{1}(t) \leq \lambda_{11}, \quad(t-a)^{n-i+1}\left|p_{i}(t)\right| \leq \lambda_{1 i} \quad(i=2, \ldots, m)
$$


hold almost everywhere on $] a, t_{0}[$, and the inequalities

$$
\begin{gathered}
(-1)^{n-m}(t-a)^{n-2 m}(b-t)^{2 m} p_{1}(t) \leq \lambda_{21}, \\
(t-a)^{n-2 m}(b-t)^{2 m-i+1}\left|p_{i}(t)\right| \leq \lambda_{2 i} \quad(i=2, \ldots, m)
\end{gathered}
$$

hold almost everywhere on $] t_{0}, b\left[\right.$. Then for every $q \in \widetilde{L}_{2 n-2 m-2,2 m-2}^{2}(] a, b[)$ problem (1.1), (1.2) is uniquely solvable in the space $\widetilde{C}^{n-1, m}(] a, b[)$.

THeOREM 1.11. Let there exist nonnegative numbers $\ell_{i}(i=1, \ldots, m)$ such that along with (1.25) the conditions

$$
(t-a)^{2 m-i} h_{i}(t, \tau) \leq \ell_{i} \quad \text { for } a<t \leq \tau \leq b(i=1, \ldots, m)
$$

hold. Then for every $\left.\left.q \in \widetilde{L}_{2 n-2 m-2}^{2}(] a, b\right]\right)$ problem (1.1), (1.3) is uniquely solvable in the space $\left.\left.\widetilde{C}^{n-1, m}(] a, b\right]\right)$.

Corollary 1.12. Let there exist nonnegative numbers $\lambda_{i}(i=1, \ldots, m)$ such that condition (1.27) holds, and the inequalities

$$
(-1)^{n-m}(t-a)^{n} p_{1}(t) \leq \lambda_{1}, \quad(t-a)^{n-i+1}\left|p_{i}(t)\right| \leq \lambda_{1 i} \quad(i=2, \ldots, m)
$$

are fulfilled almost everywhere on $] a, b\left[\right.$. Then for every $\left.\left.q \in \widetilde{L}_{2 n-2 m-2}^{2}(] a, b\right]\right)$ problem (1.1), (1.3) is uniquely solvable in the space $\left.\left.\widetilde{C}^{n-1, m}(] a, b\right]\right)$.

Remark 1.13. The above-given conditions on the unique solvability of problems (1.1), (1.2) and (1.1), (1.3) are optimal since, as Example 1.8 shows, in Theorems 1.9, 1.11 and Corollaries 1.10, 1.12 none of strict inequalities (1.21), (1.23), (1.25), and (1.27) can be replaced by nonstrict ones.

Remark 1.14. If along with the conditions of Theorem 1.9 (of Theorem 1.11) conditions (1.28) are satisfied as well, then for every $q \in \widetilde{L}_{2 n-2 m-2, m-2}^{2}(] a, b[$ ) (for every $q \in$ $\left.\left.\left.\tilde{L}_{2 n-2 m-2}^{2}(] a, b\right]\right)\right)$ problem $(1.1),(1.2)$ (problem (1.1), (1.3)) is uniquely solvable in the space $\widetilde{C}_{\text {loc }}^{n-1}(] a, b[)$ (in the space $\left.\left.\left.\widetilde{C}_{\text {loc }}^{n-1}(] a, b\right]\right)\right)$.

Remark 1.15. Corollaries 1.10 and 1.12 are more general than the results of paper [7] concerning unique solvability of problems (1.1), (1.2) and (1.1), (1.3).

\section{Auxiliary statements}

2.1. Lemmas on integral inequalities. Throughout this section, we assume that $-\infty<$ $t_{0}<t_{1}<+\infty$, and for any function $\left.u:\right] t_{0}, t_{1}\left[\rightarrow \mathbb{R}\right.$, by $u\left(t_{0}\right)$ and $u\left(t_{1}\right)$ we understand the right and the left limits of that function at the points $t_{0}$ and $t_{1}$.

Lemma 2.1. Let $\left.\left.u \in \widetilde{C}_{\mathrm{loc}}(] t_{0}, t_{1}\right]\right)$ and

$$
\int_{t_{0}}^{t_{1}}\left(t-t_{0}\right)^{\alpha+2} u^{\prime 2}(t) d t<+\infty
$$


where $\alpha \neq-1$. If, moreover, either

$$
\alpha>-1, \quad u\left(t_{1}\right)=0
$$

or

$$
\alpha<-1, \quad u\left(t_{0}\right)=0
$$

then

$$
\int_{t_{0}}^{t_{1}}\left(t-t_{0}\right)^{\alpha} u^{2}(t) d t \leq \frac{4}{(1+\alpha)^{2}} \int_{t_{0}}^{t_{1}}\left(t-t_{0}\right)^{\alpha+2} u^{\prime 2}(t) d t
$$

Proof. According to the formula of integration by parts, we have

$$
\begin{aligned}
\int_{s}^{t_{1}}\left(t-t_{0}\right)^{\alpha} u^{2}(t) d t= & \frac{1}{1+\alpha}\left[\left(t_{1}-t_{0}\right)^{1+\alpha} u^{2}\left(t_{1}\right)-\left(s-t_{0}\right)^{1+\alpha} u^{2}(s)\right] \\
& -\frac{2}{1+\alpha} \int_{s}^{t_{1}}\left(t-t_{0}\right)^{1+\alpha} u(t) u^{\prime}(t) d t \text { for } t_{0}<s<t_{1} .
\end{aligned}
$$

However,

$$
\begin{aligned}
-\frac{2}{1+\alpha}\left(t-t_{0}\right)^{1+\alpha} u(t) u^{\prime}(t) & =\left(-\frac{2}{1+\alpha}\left(t-t_{0}\right)^{1+\alpha / 2} u^{\prime}(t)\right)\left(\left(t-t_{0}\right)^{\alpha / 2} u(t)\right) \\
& \leq \frac{2}{(1+\alpha)^{2}}\left(t-t_{0}\right)^{\alpha+2} u^{\prime 2}(t)+\frac{1}{2}\left(t-t_{0}\right)^{\alpha} u^{2}(t) .
\end{aligned}
$$

Thus identity (2.5) implies

$$
\begin{aligned}
\int_{s}^{t_{1}}\left(t-t_{0}\right)^{\alpha} u^{2}(t) d t \leq & \frac{2}{1+\alpha}\left[\left(t_{1}-t_{0}\right)^{1+\alpha} u^{2}\left(t_{1}\right)-\left(s-t_{0}\right)^{1+\alpha} u^{2}(s)\right] \\
& +\frac{4}{(1+\alpha)^{2}} \int_{s}^{t_{1}}\left(t-t_{0}\right)^{\alpha+2} u^{\prime 2}(t) d t \quad \text { for } t_{0}<s<t_{1} .
\end{aligned}
$$

If conditions (2.2) are fulfilled, then in view of (2.1), (2.7) results in (2.4).

It remains to consider the case when conditions (2.3) hold. Then due to (2.1) we have

$$
\begin{gathered}
\int_{t_{0}}^{t_{1}}\left|u^{\prime}(t)\right| d t<+\infty, \\
|u(s)| \leq \int_{t_{0}}^{s}\left|u^{\prime}(t)\right| d t=\int_{t_{0}}^{s}\left(t-t_{0}\right)^{-\alpha / 2-1}\left(t-t_{0}\right)^{1+\alpha / 2}\left|u^{\prime}(t)\right| d t \\
\leq\left(\int_{t_{0}}^{s}\left(t-t_{0}\right)^{-\alpha-2} d t\right)^{1 / 2}\left(\int_{t_{0}}^{s}\left(t-t_{0}\right)^{2+\alpha} u^{\prime 2}(t) d t\right)^{1 / 2} \\
\leq|1+\alpha|^{-1 / 2}\left(s-t_{0}\right)^{-(\alpha+1) / 2}\left(\int_{t_{0}}^{s}\left(t-t_{0}\right)^{2+\alpha} u^{\prime 2}(t) d t\right)^{1 / 2} \text { for } t_{0}<s<t_{1}
\end{gathered}
$$

and, consequently,

$$
\lim _{s \rightarrow t_{0}}\left(s-t_{0}\right)^{\alpha+1} u^{2}(s)=0 .
$$


12 Linear BVPs with strong singularities

On the other hand, from (2.7) we have

$$
\begin{aligned}
\int_{s}^{t_{1}}\left(t-t_{0}\right)^{\alpha} u^{2}(t) d t \leq \frac{2}{|1+\alpha|}\left(s-t_{0}\right)^{1+\alpha} u^{2}(s) \\
+\frac{4}{(1+\alpha)^{2}} \int_{s}^{t_{1}}\left(t-t_{0}\right)^{\alpha+2} u^{\prime 2}(t) d t \quad \text { for } t_{0}<s<t_{1} .
\end{aligned}
$$

If in this inequality we pass to the limit as $s \rightarrow t_{0}$, then we get inequality (2.4).

Lemma 2.2. Let $\left.\left.u \in \widetilde{C}_{\mathrm{loc}}(] t_{0}, t_{1}\right]\right)$ and

$$
\int_{t_{0}}^{t_{1}}\left(t-t_{0}\right)^{(\alpha+1) / 2}\left|u^{\prime}(t)\right| d t<+\infty
$$

where $\alpha \neq-1$. If, moreover, either conditions (2.2) or conditions (2.3) hold, then

$$
\int_{t_{0}}^{t_{1}}\left(t-t_{0}\right)^{\alpha} u^{2}(t) d t \leq \frac{1}{|1+\alpha|}\left(\int_{t_{0}}^{t_{1}}\left(t-t_{0}\right)^{(\alpha+1) / 2}\left|u^{\prime}(t)\right| d t\right)^{2}
$$

Proof. If conditions (2.2) hold, then from identity (2.5) we find

$$
\begin{aligned}
\int_{s}^{t_{1}}\left(t-t_{0}\right)^{\alpha} u^{2}(t) d t & \leq \frac{2}{1+\alpha} \int_{s}^{t_{1}}\left(t-t_{0}\right)^{1+\alpha}\left|u^{\prime}(t)\right||u(t)| d t \\
& =\frac{2}{1+\alpha} \int_{s}^{t_{1}}\left(t-t_{0}\right)^{1+\alpha}\left|u^{\prime}(t)\right|\left|\int_{t}^{t_{1}} u^{\prime}(\tau) d \tau\right| d t \\
& \leq \frac{2}{1+\alpha} \int_{s}^{t_{1}}\left(t-t_{0}\right)^{(1+\alpha) / 2}\left|u^{\prime}(t)\right|\left(\int_{t}^{t_{1}}\left(\tau-t_{0}\right)^{(1+\alpha) / 2}\left|u^{\prime}(\tau)\right| d \tau\right) d t \\
& =\frac{1}{1+\alpha}\left(\int_{s}^{t_{1}}\left(\tau-t_{0}\right)^{(1+\alpha) / 2}\left|u^{\prime}(\tau)\right| d \tau\right)^{2} \quad \text { for } t_{0}<t<t_{1} .
\end{aligned}
$$

Consequently, inequality (2.12) is valid.

Now we consider the case where conditions (2.3) hold. Then, taking into account (2.11), we obtain

$$
|u(s)| \leq \int_{t_{0}}^{s}\left|u^{\prime}(t)\right| d t \leq\left(s-t_{0}\right)^{-(1+\alpha) / 2} \int_{t_{0}}^{s}\left(t-t_{0}\right)^{(1+\alpha) / 2}\left|u^{\prime}(t)\right| d t \quad \text { for } t_{0}<s<t_{1} .
$$


Hence it is obvious that $u$ satisfies equality (2.9). On the other hand, (2.5) yields

$$
\begin{aligned}
\int_{s}^{t_{1}}\left(t-t_{0}\right)^{\alpha} u^{2}(t) d t \leq & \frac{1}{|1+\alpha|}\left(s-t_{0}\right)^{1+\alpha} u^{2}(s) \\
& +\frac{2}{|1+\alpha|} \int_{s}^{t_{1}}\left(t-t_{0}\right)^{(1+\alpha) / 2}\left|u^{\prime}(t)\right|\left(\int_{t_{0}}^{t}\left(\tau-t_{0}\right)^{(1+\alpha) / 2}\left|u^{\prime}(\tau)\right| d \tau\right) d t \\
\leq & \frac{1}{|1+\alpha|}\left(s-t_{0}\right)^{1+\alpha} u^{2}(s) \\
& +\frac{1}{|1+\alpha|}\left(\int_{t_{0}}^{t_{1}}\left(\tau-t_{0}\right)^{(1+\alpha) / 2}\left|u^{\prime}(\tau)\right| d \tau\right)^{2} \quad \text { for } t_{0}<s<t_{1} .
\end{aligned}
$$

If in this inequality we pass to the limit as $s \rightarrow t_{0}$, then we obtain inequality (2.12).

Lemma 2.3. Let $\alpha>-1$ and

$$
y \in L_{\alpha+2,0}^{2}(] t_{0}, t_{1}[) \quad\left(y \in L_{(1+\alpha) / 2,0}(] t_{0}, t_{1}[)\right) .
$$

Then $\left.\left.y \in \widetilde{L}_{\alpha}^{2}(] t_{0}, t_{1}\right]\right)$ and

$$
\|y\|_{\tilde{L}_{\alpha}^{2}} \leq \frac{2}{1+\alpha}\|y\|_{L_{\alpha+2,0}^{2}} \quad\left(\|y\|_{\tilde{L}_{\alpha}^{2}} \leq(1+\alpha)^{-1 / 2}\|y\|_{L_{(1+\alpha) / 2,0}^{2}}\right) .
$$

Proof. By Lemma 2.1 (Lemma 2.2) and conditions (2.16), we have

$$
\begin{gathered}
\int_{t_{0}}^{s}\left(t-t_{0}\right)^{\alpha}\left(\int_{t}^{s} y(\tau) d \tau\right)^{2} d t \leq \frac{4}{(1+\alpha)^{2}} \int_{t_{0}}^{s}\left(t-t_{0}\right)^{\alpha+2} y^{2}(t) d t \quad \text { for } t_{0} \leq s \leq t_{1} \\
\left(\int_{t_{0}}^{s}\left(t-t_{0}\right)^{\alpha}\left(\int_{t}^{s} y(\tau) d \tau\right)^{2} d t \leq \frac{1}{1+\alpha}\left(\int_{t_{0}}^{s}\left(t-t_{0}\right)^{(1+\alpha) / 2}|y(t)| d t\right)^{2} \text { for } t_{0} \leq s \leq t_{1}\right),
\end{gathered}
$$

which guarantees the validity of inequality (2.17).

The following lemma easily follows from Lemma 2.3.

LEMMA 2.4. Let $\alpha>-1, \beta>-1$, and

$$
y \in L_{\alpha+2, \beta+2}^{2}(] t_{0}, t_{1}[) \quad\left(y \in L_{(1+\alpha) / 2,(1+\beta) / 2}(] t_{0}, t_{1}[)\right) .
$$

Then $y \in \tilde{L}_{\alpha, \beta}^{2}(] t_{0}, t_{1}[)$ and

$$
\|y\|_{\tilde{L}_{\alpha, \beta}^{2}} \leq \gamma\|y\|_{L_{\alpha+2, \beta+2}^{2}} \quad\left(\|y\|_{\widetilde{L}_{\alpha, \beta}^{2}} \leq \gamma\|y\|_{L_{(1+\alpha) / 2,(1+\beta) / 2}}\right),
$$

where

$$
\begin{gathered}
\gamma=\frac{2}{1+\alpha}\left(\frac{2}{t_{1}-t_{0}}\right)^{1+\beta / 2}+\frac{2}{1+\beta}\left(\frac{2}{t_{1}-t_{0}}\right)^{1+\alpha / 2} \\
\left(\gamma=(1+\alpha)^{-1 / 2}\left(\frac{2}{t_{1}-t_{0}}\right)^{(1+\beta) / 2}+(1+\beta)^{-1 / 2}\left(\frac{2}{t_{1}-t_{0}}\right)^{(1+\alpha) / 2}\right) .
\end{gathered}
$$


14 Linear BVPs with strong singularities

LemmA 2.5. Let $u \in \widetilde{C}_{\mathrm{loc}}^{m-1}(] t_{0}, t_{1}[)$,

$$
u^{(i-1)}\left(t_{0}\right)=0 \quad(i=1, \ldots, m), \quad \int_{t_{0}}^{t_{1}}\left|u^{(m)}(t)\right|^{2} d t<+\infty .
$$

Then

$$
\int_{t_{0}}^{t_{1}} \frac{u^{2}(t)}{\left(t-t_{0}\right)^{2 m}} d t \leq\left(\frac{2^{m}}{(2 m-1) ! !}\right)^{2} \int_{t_{0}}^{t_{1}}\left|u^{(m)}(t)\right|^{2} d t
$$

Proof. By virtue of Lemma 2.1 and conditions (2.22), we have

$$
\int_{t_{0}}^{t_{1}} \frac{\left|u^{(i-1)}(t)\right|^{2}}{\left(t-t_{0}\right)^{2 m-2 i+2}} d t \leq \frac{4}{(2 m-2 i+1)^{2}} \int_{t_{0}}^{t_{1}} \frac{\left|u^{(i)}(t)\right|^{2}}{\left(t-t_{0}\right)^{2 m-2 i}} d t<+\infty \quad(i=1, \ldots, m) .
$$

The inequality (2.23) is now immediate.

Remark 2.6. Inequality (2.23) cannot be replaced by the inequality

$$
\int_{t_{0}}^{t_{1}} \frac{u^{2}(t)}{\left(t-t_{0}\right)^{2 m}} d t \leq\left[\left(\frac{2^{m}}{(2 m-1) ! !}\right)^{2}-\varepsilon\right] \int_{t_{0}}^{t_{1}}\left|u^{(m)}(t)\right|^{2} d t
$$

no matter how small $\varepsilon>0$. Indeed, choose $\delta \in] 0,1[$ so small that

$$
2^{2 m} \prod_{i=1}^{m}(2 i-1-\delta)^{-2}>\left(\frac{2^{m}}{(2 m-1) ! !}\right)^{2}-\varepsilon
$$

Then the function $u(t)=(t-a)^{m-(1-\delta) / 2}$ satisfies conditions (2.22) but inequality $(2.25)$ is violated.

From Lemma 2.5, by the change of variable, we obtain the following lemma.

Lemma $2.5^{\prime}$. Let $u \in \widetilde{C}_{\mathrm{loc}}^{m-1}(] t_{0}, t_{1}[)$,

$$
u^{(i-1)}\left(t_{1}\right)=0 \quad(i=1, \ldots, m), \quad \int_{t_{0}}^{t_{1}}\left|u^{(m)}(t)\right|^{2} d t<+\infty .
$$

Then

$$
\int_{t_{0}}^{t_{1}} \frac{u^{2}(t)}{\left(t-t_{1}\right)^{2 m}} d t \leq\left(\frac{2^{m}}{(2 m-1) ! !}\right)^{2} \int_{t_{0}}^{t_{1}}\left|u^{(m)}(t)\right|^{2} d t
$$

LeMmA 2.7. Let $u \in \widetilde{C}_{\mathrm{loc}}^{m-1}(] t_{0}, t_{1}[)$ be a function satisfying conditions $(2.22)$, and $p \in$ $\left.\left.L_{\mathrm{loc}}(] t_{0}, t_{1}\right]\right)$ be such that

$$
\left(t-t_{0}\right)^{2 m-j}\left|\int_{t}^{t_{1}} p(\tau) d \tau\right| \leq \ell_{0} \quad \text { for } t_{0}<t \leq t_{1}
$$


where $j \in\{1, \ldots, m\}$ and $\ell_{0}>0$. Then

$$
\left|\int_{t}^{t_{1}} p(s) u(s) u^{(j-1)}(s) d s\right| \leq \ell_{0}\left[\rho(t)+\frac{(2 m-j) 2^{2 m-j+1}}{(2 m-1) ! !(2 m-2 j+1) ! !} \rho\left(t_{1}\right)\right] \quad \text { for } t_{0}<t \leq t_{1},
$$

where

$$
\rho(t)=\int_{t_{0}}^{t}\left|u^{(m)}(s)\right|^{2} d s .
$$

Proof. In view of the formula of integration by parts, we have

$$
\int_{t}^{t_{1}} p(s) u(s) u^{(j-1)}(s) d s=u(t) u^{(j-1)}(t) \int_{t}^{t_{1}} p(\tau) d \tau+\sum_{k=0}^{1} \int_{t}^{t_{1}}\left(\int_{s}^{t_{1}} p(\tau) d \tau\right) u^{(k)}(s) u^{(j-k)}(s) d s .
$$

On the other hand, by conditions (2.22), the Schwartz inequality, and Lemma 2.5, it follows that

$$
\begin{aligned}
\left|u^{(i-1)}(t)\right| & =\frac{1}{(m-i) !}\left|\int_{t_{0}}^{t}(t-s)^{m-i} u^{(m)}(s) d s\right| \\
& \leq\left(t-t_{0}\right)^{m-i+1 / 2} \rho^{1 / 2}(t) \quad \text { for } t_{0}<t \leq t_{1}(i=1, \ldots, m), \\
\int_{t_{0}}^{t_{1}} \frac{\left|u^{(i-1)}(s)\right|^{2}}{(s-a)^{2 m-2 i+2}} d s & \leq \frac{2^{m-i+1}}{(2 m-2 i+1) ! !} \rho^{1 / 2}\left(t_{1}\right) \quad(i=1, \ldots, m) .
\end{aligned}
$$

If along with this we take into account inequality (2.29), we obtain

$$
\begin{aligned}
& \left|\int_{t}^{t_{1}} p(s) u(s) u^{(j-1)}(s) d s\right| \\
& \quad \leq \ell_{0} \rho(t)+\ell_{0} \sum_{k=0}^{1} \int_{t}^{t_{1}}\left(s-t_{0}\right)^{2 m-j}\left|u^{(k)}(s) u^{(j-k)}(s)\right| d s \\
& \quad \leq \ell_{0} \rho(t)+\ell_{0} \sum_{k=0}^{1}\left(\int_{t}^{t_{1}} \frac{\left|u^{(k)}(s)\right|^{2} d s}{(s-a)^{2 m-2 k}}\right)^{1 / 2}\left(\int_{t}^{t_{1}} \frac{\left|u^{(j-k)}(s)\right|^{2} d s}{(s-a)^{2 m+2 k-2 j}}\right)^{1 / 2} \\
& \quad \leq \ell_{0} \rho(t)+\ell_{0} \rho\left(t_{1}\right) \sum_{k=0}^{1} \frac{2^{2 m-j}}{(2 m-2 k-1) ! !(2 m-2 j+2 k-1) ! !} \text { for } t_{0}<t \leq t_{1} .
\end{aligned}
$$

Therefore, estimate (2.30) is valid.

The following lemma can be proved similarly to Lemma 2.7.

Lemma $2.6^{\prime}$. Let $u \in \widetilde{C}_{\mathrm{loc}}^{m-1}(] t_{0}, t_{1}[)$ be a function satisfying conditions (2.27), and $p \in$ $L_{\mathrm{loc}}\left(\left[t_{0}, t_{1}[)\right.\right.$ be such that

$$
\left(t_{1}-t\right)^{2 m-j}\left|\int_{t_{0}}^{t} p(\tau) d \tau\right| \leq \ell_{0} \quad \text { for } t_{0} \leq t<t_{1}
$$


16 Linear BVPs with strong singularities

where $j \in\{1, \ldots, m\}$ and $\ell_{0}>0$. Then

$$
\left|\int_{t_{0}}^{t} p(s) u(s) u^{(j-1)}(s) d s\right| \leq \ell_{0}\left[\rho(t)+\frac{(2 m-j) 2^{2 m-j+1}}{(2 m-1) ! !(2 m-2 j+1) ! !} \rho\left(t_{0}\right)\right] \quad \text { for } t_{0} \leq t<t_{1},
$$

where

$$
\rho(t)=\int_{t}^{t_{1}}\left|u^{(m)}(s)\right|^{2} d s
$$

2.2. A lemma on the properties of functions from the space $\widetilde{C}^{n-1, m}(] a, b[)$. In this section, as above, we assume that $m$ is the integral part of the number $n / 2$.

LEMMA 2.8. Let

$$
w(t)=\sum_{i=1}^{n-m} \sum_{k=i}^{n-m} c_{i k}(t) u^{(n-k)}(t) u^{(i-1)}(t)
$$

where $u \in \widetilde{C}^{n-1, m}(] a, b[)$, and each $c_{i k}:[a, b] \rightarrow \mathbb{R}$ is an $(n-k-i+1)$-times continuously differentiable function. If, moreover,

$$
u^{(i-1)}(a)=0 \quad(i=1, \ldots, m), \quad \limsup _{t \rightarrow a} \frac{\left|c_{i i}(t)\right|}{(t-a)^{n-2 m}}<+\infty \quad(i=1, \ldots, n-m),
$$

then

$$
\liminf _{t \rightarrow a}|w(t)|=0
$$

and if

$$
u^{(i-1)}(b)=0 \quad(i=1, \ldots, n-m),
$$

then

$$
\liminf _{t \rightarrow b}|w(t)|=0 .
$$

The proof of this lemma is given in [12].

2.3. Lemmas on the sequences of solutions of auxiliary problems. Suppose

$$
a<t_{0 k}<t_{1 k}<b \quad(k=1,2, \ldots), \quad \lim _{k \rightarrow+\infty} t_{0 k}=a, \quad \lim _{k \rightarrow+\infty} t_{1 k}=b .
$$

For the differential equation

$$
u^{(n)}=\sum_{i=1}^{m} p_{i}(t) u^{(i-1)}+q_{k}(t)
$$


we consider the auxiliary boundary conditions

$$
\begin{array}{llll}
u^{(i-1)}\left(t_{0 k}\right)=0 & (i=1, \ldots, m), & u^{(i-1)}\left(t_{1 k}\right)=0 & (i=1, \ldots, n-m), \\
u^{(i-1)}\left(t_{0 k}\right)=0 & (i=1, \ldots, m), & u^{(i-1)}(b)=0 & (i=1, \ldots, n-m),
\end{array}
$$

for every natural $k$.

Throughout this section, when problems (1.1), (1.2) and (2.44), (2.45) are discussed, we assume that

$$
p_{i} \in L_{\mathrm{loc}}(] a, b[) \quad(i=1, \ldots, m), \quad q, q_{k} \in \widetilde{L}_{2 n-2 m-2,2 m-2}^{2}(] a, b[),
$$

and in the case $n=2 m+1$ in addition we assume the conditions

$$
\rho_{i} \stackrel{\text { def }}{=} \sup \left\{(b-t)^{2 m-i}\left|\int_{t_{1}}^{t} p_{i}(s) d s\right|: t_{0} \leq t<b\right\}<+\infty \quad(i=1, \ldots, m),
$$

where $t_{1}=(a+b) / 2$.

As for problems (1.1), (1.3) and (2.44), (2.46), they are considered in the case, where

$$
\left.\left.p_{i} \in L_{\mathrm{loc}}(] a, b\right]\right) \quad(i=1, \ldots, m), \quad q, q_{k} \in \tilde{L}_{2 n-2 m-2,0}^{2}(] a, b[) .
$$

LEMMA 2.9. Let for every natural $k$, problem (2.44), (2.45) have a solution $u_{k} \in \widetilde{C}_{\mathrm{loc}}^{n-1}(] a$, $b[)$, and there exist a nonnegative constant $r_{0}$ such that

$$
\int_{t_{0 k}}^{t_{1 k}}\left|u_{k}^{(m)}(t)\right|^{2} d t \leq r_{0}^{2} \quad(k=1,2, \ldots)
$$

Let, moreover,

$$
\lim _{k \rightarrow+\infty}\left\|q_{k}-q\right\|_{\tilde{L}_{2 n-2 m-2,2 m-2}^{2}}=0
$$

and the homogeneous problem (1.1 $)$, (1.2) have only a trivial solution in the space $\widetilde{C}^{n-1, m}(] a, b[)$. Then problem (1.1), (1.2) has a unique solution $u$ such that

$$
\begin{gathered}
\left\|u^{(m)}\right\|_{L^{2}} \leq r_{0} \\
\left.\lim _{k \rightarrow+\infty} u_{k}^{(i-1)}(t)=u^{(i-1)}(t) \quad(i=1, \ldots, n) \text { uniformly in }\right] a, b[.
\end{gathered}
$$

(That is, uniformly on $[a+\delta, b-\delta]$ for an arbitrarily small $\delta>0$ ).

Proof. For an arbitrary $(m-1)$-times continuously differentiable function $v:] a, b[\rightarrow \mathbb{R}$, we set

$$
\Lambda(v)(t)=\sum_{i=1}^{m} p_{i}(t) v^{(i-1)}(t)
$$

Suppose $t_{1}, \ldots, t_{n}$ are the numbers such that

$$
(a+b) / 2=t_{1}<\cdots<t_{n}<b,
$$


and $g_{i}(t)(i=1, \ldots, n)$ are the polynomials of $(n-1)$ th degree, satisfying the conditions

$$
g_{i}\left(t_{i}\right)=1, \quad g_{i}\left(t_{j}\right)=0 \quad(i \neq j ; i, j=1, \ldots, n) .
$$

Then for every natural $k$, the representation

$$
\begin{aligned}
u_{k}(t)= & \sum_{j=1}^{n}\left(u_{k}\left(t_{j}\right)-\frac{1}{(n-1) !} \int_{t_{1}}^{t_{j}}\left(t_{j}-s\right)^{n-1}\left(\Lambda\left(u_{k}\right)(s)+q_{k}(s)\right) d s\right) g_{j}(t) \\
& +\frac{1}{(n-1) !} \int_{t_{1}}^{t}(t-s)^{n-1}\left(\Lambda\left(u_{k}\right)(s)+q_{k}(s)\right) d s
\end{aligned}
$$

is valid.

For an arbitrary $\delta \in] 0,(b-a) / 2[$, we have

$$
\begin{aligned}
& \left|\int_{t_{1}}^{t}(t-s)^{n-i}\left(q_{k}(s)-q(s)\right) d s\right| \\
& \quad=(n-i)\left|\int_{t_{1}}^{t}(t-s)^{n-i-1}\left(\int_{t_{1}}^{s}\left(q_{k}(\tau)-q(\tau)\right) d \tau\right) d s\right| \\
& \quad \leq n \int_{a+\delta}^{t_{1}}(s-a)^{m-i}(s-a)^{n-m-1}\left|\int_{s}^{t_{1}}\left(q_{k}(\tau)-q(\tau)\right) d \tau\right| d s \\
& \quad \leq n\left(\int_{a+\delta}^{t_{1}}(s-a)^{2 m-2 i} d s\right)^{1 / 2}\left(\int_{a+\delta}^{t_{1}}(s-a)^{2 n-2 m-2}\left|\int_{s}^{t_{1}}\left(q_{k}(\tau)-q(\tau)\right) d \tau\right|^{2} d s\right)^{1 / 2} \\
& \quad \leq n\left|\left(t_{1}-a\right)^{2 m-2 i+1}-\delta^{2 m-2 i+1}\right|^{1 / 2}|| q_{k}-q \|\left.\right|_{\tilde{L}_{2 n-2 m-2,2 m-2}^{2}} \\
& \quad \text { for } a+\delta \leq t \leq t_{1}(i=1, \ldots, n-1), \\
& \left|\int_{t_{1}}^{t}(t-s)^{n-i}\left(q_{k}(s)-q(s)\right) d s\right| \leq n\left|\left(b-t_{1}\right)^{2 n-2 m-2 i+1}-\delta^{2 n-2 m-2 i+1}\right|^{1 / 2}\left\|q_{k}-q\right\|_{\tilde{L}_{2 n-2 m-2,2 m-2}^{2}} \\
& \text { for } t_{1} \leq t \leq b-\delta(i=1, \ldots, n-1) .
\end{aligned}
$$

Hence, by condition (2.51), we find

$$
\left.\lim _{k \rightarrow+\infty} \int_{t_{1}}^{t}(t-s)^{n-i}\left(q_{k}(s)-q(s)\right) d s=0 \quad(i=1, \ldots, n) \text { uniformly in }\right] a, b[.
$$

Analogously we can show that if $\left.t_{0} \in\right] a, b[$, then

$$
\lim _{k \rightarrow+\infty} \int_{t_{0}}^{t}\left(s-t_{0}\right)\left(q_{k}(s)-q(s)\right) d s=0 \text { uniformly on } I\left(t_{0}\right)
$$

where $I\left(t_{0}\right)=\left[t_{0},(a+b) / 2\right]$ for $t_{0}<(a+b) / 2$ and $I\left(t_{0}\right)=\left[(a+b) / 2, t_{0}\right]$ for $t_{0}>(a+b) / 2$.

In view of inequalities (2.50), the identities

$$
u_{k}^{(i-1)}(t)=\frac{1}{(m-i) !} \int_{t_{j k}}^{t}(t-s)^{m-i} u_{k}^{(m)}(s) d s \quad(j=0,1 ; i=1, \ldots, m ; k=1,2, \ldots)
$$


yield

$$
\left|u_{k}^{(i-1)}(t)\right| \leq r_{i}[(t-a)(b-t)]^{m-i+1 / 2} \text { for } t_{1 k} \leq t \leq t_{2 k}(i=1, \ldots, m ; k=1,2, \ldots),
$$

where

$$
r_{i}=\frac{r_{0}}{(m-i) !}(2 m-2 i+1)^{-1 / 2}\left(\frac{2}{b-a}\right)^{m-i+1 / 2} \quad(i=1, \ldots, m)
$$

By virtue of the Arzela-Ascoli lemma and conditions (2.50), (2.62), the sequence $\left(u_{k}\right)_{k=1}^{+\infty}$ contains a subsequence $\left(u_{k_{\ell}}\right)_{\ell=1}^{+\infty}$ such that $\left(u_{k_{\ell}}^{(i-1)}\right)_{\ell=1}^{+\infty}(i=1, \ldots, m)$ are uniformly converging on $] a, b[$. Suppose

$$
\lim _{\ell \rightarrow+\infty} u_{k_{\ell}}(t)=u(t)
$$

Then $u:] a, b[\rightarrow \mathbb{R}$ is $(m-1)$-times continuously differentiable and

$$
\left.\lim _{\ell \rightarrow+\infty} u_{k_{\ell}}^{(i-1)}(t)=u^{(i-1)}(t) \quad(i=1, \ldots, m) \text { uniformly on }\right] a, b[
$$

If along with this we take into account conditions (2.43) and (2.59), then from (2.57) and (2.62) we find

$$
\begin{gathered}
u(t)=\sum_{j=1}^{n}\left(u\left(t_{j}\right)-\frac{1}{(n-1) !} \int_{t_{1}}^{t_{j}}\left(t_{j}-s\right)^{n-1}(\Lambda(u)(s)+q(s)) d s\right) g_{j}(t) \\
\quad+\frac{1}{(n-1) !} \int_{t_{1}}^{t}(t-s)^{n-1}(\Lambda(u)(s)+q(s)) d s \quad \text { for } a<t<b, \\
\left|u^{(i-1)}(t)\right| \leq r_{i}[(t-a)(b-t)]^{m-i+1 / 2} \quad \text { for } a<t<b(i=1, \ldots, m),
\end{gathered}
$$

$u \in \widetilde{C}_{\mathrm{loc}}^{n-1}(] a, b[)$, and

$$
\left.\lim _{\ell \rightarrow+\infty} u_{k_{\ell}}^{(i-1)}(t)=u^{(i-1)}(t) \quad(i=1, \ldots, n-1) \text { uniformly in }\right] a, b[
$$

On the other hand, for any $\left.t_{0} \in\right] a, b$ [ and a natural $\ell$, we have

$$
\left(t-t_{0}\right) u_{k_{\ell}}^{(n-1)}(t)=u_{k_{\ell}}^{(n-2)}(t)-u_{k_{\ell}}^{(n-2)}\left(t_{0}\right)+\int_{t_{0}}^{t}\left(s-t_{0}\right)\left(\Lambda\left(u_{k_{\ell}}\right)(s)+q_{k_{\ell}}(s)\right) d s
$$

Hence, due to (2.60) and (2.68), we get

$$
\left.\lim _{\ell \rightarrow+\infty} u_{k_{\ell}}^{(n-1)}(t)=u^{(n-1)}(t) \text { uniformly in }\right] a, b[\text {. }
$$


By (2.68) and (2.70), (2.50) results in (2.52). Therefore, $u \in \widetilde{C}^{n-1, m}([a, b[)$. On the other hand, from (2.66) it is obvious that $u$ is a solution of (1.1). In the case, where $n=2 m$, from (2.67) equalities (1.2) follow, that is, $u$ is a solution of problem (1.1), (1.2).

Let us show that $u$ is a solution of that problem in the case $n=2 m+1$ as well. In view of (2.67), it suffices to prove that $u^{(m)}(b)=0$. First we find an estimate for the sequence $\left(u_{k}^{(m+1)}\right)_{k=1}^{+\infty}$. For this, without loss of generality we assume that

$$
t_{1}<t_{1 k} \quad(k=1,2, \ldots)
$$

By (2.51), (2.57), and (2.62), we have

$$
\begin{gathered}
\left|u_{k}^{(m+1)}(t)\right| \leq \rho_{0}+\frac{1}{(m-1) !}\left|\int_{t_{1}}^{t}(t-s)^{m-1} \Lambda\left(u_{k}\right)(s) d s\right|+\frac{1}{(m-1) !}\left|\int_{t_{1}}^{t}(t-s)^{m-1} q_{k}(s) d s\right| \\
\quad \text { for } t_{1} \leq t \leq t_{1 k}(k=1,2, \ldots) \\
\left\|q_{k}\right\|_{\tilde{L}_{2 n-2 m-2,2 m-2}^{2}} \leq \rho_{0} \quad(k=1,2, \ldots),
\end{gathered}
$$

where $\rho_{0}$ is a positive constant independent on $k$. On the other hand, it is evident that

$$
\left|\int_{t_{1}}^{t}(t-s)^{m-1} \Lambda\left(u_{k}\right)(s) d s\right| \leq \sum_{i=1}^{m}\left|\int_{t_{1}}^{t}(t-s)^{m-1} p_{i}(s) u_{k}^{(i-1)}(s) d s\right| .
$$

If $m>1$, then in view of $(2.48)$ we find

$$
\begin{aligned}
& \left|\int_{t_{1}}^{t}(t-s)^{m-1} p_{i}(s) u_{k}^{(i-1)}(s) d s\right| \\
& \quad=\left|\int_{t_{1}}^{t}\left[(t-s)^{m-1} u_{k}^{(i)}(s)-(m-1)(t-s)^{m-2} u_{k}^{(i-1)}(s)\right]\left(\int_{t_{1}}^{s} p_{i}(\tau) d \tau\right) d s\right| \\
& \quad \leq \rho_{i} \int_{t_{1}}^{t}\left[(b-s)^{i-m-1}\left|u_{k}^{(i)}(s)\right|+(m-1)(b-s)^{i-m-2}\left|u_{k}^{(i-1)}(s)\right|\right] d s \\
& \quad \leq \rho_{i}\left(\int_{t_{1}}^{t}(b-s)^{-2} d s\right)^{1 / 2}\left[\left(\int_{t_{1}}^{t} \frac{\left|u_{k}^{(i)}(s)\right|^{2} d s}{(b-s)^{2 m-2 i}}\right)^{1 / 2}+(m-1)\left(\int_{t_{1}}^{t} \frac{\left|u_{k}^{(i-1)}(s)\right|^{2} d s}{(b-s)^{2 m-2 i+2}}\right)^{1 / 2}\right] \\
& \text { for } t_{1} \leq t \leq t_{1 k}(i=1, \ldots, m) .
\end{aligned}
$$

However, by Lemma $2.5^{\prime}$ and conditions (2.50),

$$
\int_{t_{1}}^{t} \frac{\left|u_{k}^{(j)}(s)\right|^{2} d s}{(b-s)^{2 m-2 j}} \leq \int_{t_{1}}^{t_{1 k}} \frac{\left|u_{k}^{(j)}(s)\right|^{2} d s}{\left(t_{1 k}-s\right)^{2 m-2 j}} \leq 2^{2 m-2 j} r_{0}^{2} \quad \text { for } t_{1} \leq t \leq t_{1 k}(j=0, \ldots, m) .
$$

Thus

$$
\left|\int_{t_{1}}^{t}(t-s)^{m-1} \Lambda\left(u_{k}\right)(s) d s\right| \leq \rho(b-t)^{-1 / 2} \text { for } t_{1} \leq t \leq t_{1 k}
$$


where

$$
\rho=m 2^{m} r_{0} \sum_{i=1}^{m} \rho_{i}
$$

And if $m=1$, then due to (2.48) and (2.50) we obtain

$$
\begin{aligned}
& \left|\int_{t_{1}}^{t}(t-s)^{m-1} \Lambda\left(u_{k}\right)(s) d s\right| \\
& \quad=\left|\int_{t_{1}}^{t} p_{1}(s) u_{k}(s) d s\right| \\
& \quad=\left|u_{k}(t) \int_{t_{1}}^{t} p_{1}(\tau) d \tau-\int_{t_{1}}^{t}\left(\int_{t_{1}}^{s} p_{1}(\tau) d \tau\right) u_{k}^{\prime}(s) d s\right| \\
& \quad \leq \rho_{1}\left((b-t)^{-1} \int_{t}^{t_{1 k}}\left|u_{k}^{\prime}(s)\right| d s+\int_{t_{1}}^{t}(b-s)^{-1}\left|u_{k}^{\prime}(s)\right| d s\right) \\
& \quad \leq \rho_{1}\left[(b-t)^{-1}\left(t_{1 k}-t\right)^{1 / 2}\left(\int_{t}^{t_{1 k}}\left|u_{k}^{\prime}(s)\right|^{2} d s\right)^{1 / 2}+(b-t)^{-1 / 2}\left(\int_{t_{1}}^{t}\left|u_{k}^{\prime}(s)\right|^{2} d s\right)^{1 / 2}\right] \\
& \quad \leq 2 \rho_{1} r_{0}(b-t)^{-1 / 2} \quad \text { for } t_{1} \leq t \leq t_{1 k},
\end{aligned}
$$

that is, again estimate (2.77) is valid.

For $m>1$, due to condition (2.73) we have

$$
\begin{aligned}
\left|\int_{t_{1}}^{t}(t-s)^{m-1} q_{k}(s) d s\right| & =(m-1)\left|\int_{t_{1}}^{t}(t-s)^{m-2}\left(\int_{t_{1}}^{s} q_{k}(\tau) d \tau\right) d s\right| \\
& \leq(m-1) \int_{t_{1}}^{t}(b-s)^{m-2}\left(\int_{t_{1}}^{s}\left|q_{k}(\tau)\right| d \tau\right) d s \\
& \leq(m-1)(b-t)^{-1 / 2}|| q_{k} \|_{\tilde{L}_{2 n-2 m-2,2 m-2}^{2}} \\
& \leq(m-1) \rho_{0}(b-t)^{-1 / 2} \text { for } t_{1} \leq t<b .
\end{aligned}
$$

And for $m=1$, we have

$$
\int_{t}^{b}\left|\int_{t_{1}}^{\tau} q_{k}(s) d s\right| d \tau \leq(b-t)^{1 / 2}\|q\|_{\tilde{L}_{0,0}^{2}} \leq \rho_{0}(b-t)^{1 / 2} \quad \text { for } t_{1} \leq t<b .
$$

Evidently,

$$
u_{k}^{(m)}(t)=\int_{t_{1 k}}^{t} u_{k}^{(m+1)}(\tau) d \tau
$$

since $u_{k}^{(m)}\left(t_{1 k}\right)=0$. If $m>1$, then from (2.82), on account of inequalities (2.72), (2.77), and (2.80), we get

$$
\left|u_{k}^{(m)}(t)\right| \leq \int_{t}^{t_{1 k}}\left[\rho_{0}+\left(\rho+\rho_{0}\right)(b-s)^{-1 / 2}\right] d s \leq \rho^{*}(b-t)^{1 / 2} \quad \text { for } t_{1} \leq t \leq t_{1 k},
$$


where $\rho^{*}=\rho_{0}\left(b-t_{1}\right)^{1 / 2}+2\left(\rho+\rho_{0}\right)$. If $m=1$, then by virtue of inequalities $(2.72),(2.77)$, and (2.81), from (2.82) we find

$$
\begin{aligned}
\left|u_{k}^{(m)}(t)\right| & \leq \int_{t}^{t_{1 k}}\left[\rho_{0}+\rho(b-s)^{-1 / 2}+\left|\int_{t_{1}}^{s} q_{k}(\tau) d \tau\right|\right] d s \\
& \leq\left[\rho_{0}\left(b-t_{1}\right)^{1 / 2}+2 \rho+\rho_{0}\right](b-t)^{1 / 2} \quad \text { for } t_{1} \leq t \leq t_{1 k}
\end{aligned}
$$

that is, again estimate $(2.83)$ is valid.

By virtue of (2.43), (2.68) and (2.70), (2.83) implies

$$
\left|u^{(m)}(t)\right| \leq \rho^{*}(b-t)^{1 / 2} \quad \text { for } t_{1} \leq t<b,
$$

and consequently, $u^{(m)}(b)=0$. Thus we proved that $u$ is a solution of problem (1.1), (1.2) also in the case $n=2 m+1$. In the space $\widetilde{C}^{n-1, m}(] a, b[)$ problem (1.1), (1.2) does not have another solution since in that space the homogeneous problem $\left(1.1_{0}\right),(1.2)$ has only a trivial solution.

To complete the proof of the lemma, it remains to show that condition (2.53) is satisfied. Assume the contrary. Then there exist $\delta \in] 0,(b-a) / 2[, \varepsilon>0$, and an increasing sequence of natural numbers $\left(k_{\ell}\right)_{\ell=1}^{+\infty}$ such that

$$
\max \left\{\sum_{i=1}^{n}\left|u_{k_{\ell}}^{(i-1)}(t)-u^{(i-1)}(t)\right|: a+\delta \leq t \leq b-\delta\right\}>\varepsilon \quad(\ell=1,2, \ldots) .
$$

By virtue of the Arzela-Ascoli lemma and condition (2.50), the sequences $\left(u_{k_{\ell}}^{(i-1)}\right)_{\ell=1}^{+\infty}$ $(i=1, \ldots, m)$, without loss of generality, can be assumed to be uniformly converging on ]$a, b[$. Then, in view of what we have shown above, conditions (2.68) and (2.70) hold. But this contradicts condition (2.86). The obtained contradiction proves the validity of the lemma.

Analogously we can prove the following lemma.

LEMMA 2.10. Let for every natural $k$, problem (2.44), (2.46) have a solution $u_{k} \in \widetilde{C}_{\mathrm{loc}}^{n-1}$ (] a, $b]$ ), and there exist a nonnegative constant $r_{0}$ such that inequalities (2.50) are fulfilled. Let, moreover,

$$
\lim _{k \rightarrow+\infty}\left\|q_{k}-q\right\|_{\tilde{L}_{2 n-2 m-2,0}^{2}}=0
$$

and the homogeneous problem $\left(1.1_{0}\right),(1.3)$ in the space $\left.\left.\widetilde{C}^{n-1, m}(] a, b\right]\right)$ have only a trivial solution. Then problem (1.1), (1.3) in the space $\left.\left.\widetilde{C}^{n-1, m}(] a, b\right]\right)$ has a unique solution $u$, satisfying estimate (2.52) and

$$
\left.\left.\lim _{k \rightarrow+\infty} u_{k}^{(i-1)}(t)=u^{(i-1)}(t) \quad(i=1, \ldots, n) \text { uniformly in }\right] a, b\right]
$$




\subsection{Lemmas on a priori estimates.}

Lemma 2.11. Let conditions (1.20) and (1.21) be fulfilled, where $h_{i}(i=1, \ldots, m)$ are functions given by equalities (1.13), $\left.a_{0} \in\right] a, b\left[, b_{0} \in\right] a_{0}, b\left[\right.$, and $\ell_{1 i}, \ell_{2 i}(i=1, \ldots, m)$ are nonnegative numbers. Then there exists a positive constant $r_{0}$ such that for any $\left.t_{0} \in\right] a, a_{0}[$, $\left.t_{1} \in\right] b_{0}, b\left[\right.$, and $q \in \widetilde{L}_{2 n-2 m-2,2 m-2}^{2}(] a, b[)$, an arbitrary solution $u \in C_{\mathrm{loc}}^{n-1}(] a, b[)$ of (1.1), satisfying the conditions

$$
\begin{array}{ll}
u^{(i-1)}\left(t_{0}\right)=0 & (i=1, \ldots, m), \\
u^{(j-1)}\left(t_{1}\right)=0 & (j=1, \ldots, n-m),
\end{array}
$$

satisfies also the condition

$$
\int_{t_{0}}^{t_{1}}\left|u^{(m)}(t)\right|^{2} d t \leq r_{0}\left(\left|\sum_{i=1}^{m} \int_{a_{0}}^{b_{0}}(t-a)^{n-2 m} p_{i}(t) u^{(i-1)}(t) u(t) d t\right|+\|q\|_{\widetilde{L}_{2 n-2 m-2,2 m-2}^{2}}^{2}\right) .
$$

To prove Lemma 2.11, we need the following lemma.

LEMMA 2.12. If $u \in C_{\mathrm{loc}}^{n-1}(] a, b[)$, then for any $s$ and $\left.t \in\right] a, b$ [ the equality

$$
(-1)^{n-m} \int_{s}^{t}(\tau-a)^{n-2 m} u^{(n)}(\tau) u(\tau) d \tau=w_{n}(t)-w_{n}(s)+\mu_{n} \int_{s}^{t}\left|u^{(m)}(\tau)\right|^{2} d \tau
$$

is valid, where

$$
\begin{gathered}
\mu_{2 m}=1, \quad \mu_{2 m+1}=\frac{2 m+1}{2}, \quad w_{2 m}(t)=\sum_{j=1}^{m}(-1)^{m+j-1} u^{(2 m-j)}(t) u(t), \\
w_{2 m+1}(t)=\sum_{j=1}^{m}(-1)^{m+j}\left[(t-a) u^{(2 m+1-j)}(t)-j u^{(2 m-j)}(t)\right] u^{(j-1)}(t)-\frac{t-a}{2}\left|u^{(m)}(t)\right|^{2} .
\end{gathered}
$$

This lemma is a particular case of Lemma 4.1 in [8].

Proof of Lemma 2.11. By virtue of inequalities (1.21), there exists $\gamma \in] 0,1[$ such that

$$
\sum_{i=1}^{m} \frac{(2 m-i) 2^{2 m-i+1}}{(2 m-2 i+1) ! !(2 m-1) ! !} \ell_{j i}<\mu_{n}-\gamma \quad(j=1,2) .
$$

Put

$$
r_{0}=2^{2 m+2}(1+b-a)^{2} \gamma^{-2}
$$


Assume now that for some $\left.t_{0} \in\right] a, a_{0}\left[, t_{1} \in\right] b_{0}, b\left[\right.$, and $q \in \tilde{L}_{2 n-2 m-2,2 m-2}^{2}(] a, b[)$ problem (1.1), (2.89) has a solution $u$. Multiplying $(1.1)$ by $(-1)^{n-m}(t-a)^{n-2 m} u(t)$ and then integrating from $t_{0}$ to $t_{1}$, by Lemma 2.12 we obtain

$$
\begin{aligned}
\frac{t_{0}-a}{2}\left|u^{(m)}\left(t_{0}\right)\right|^{2}+\mu_{n} \int_{t_{0}}^{t_{1}}\left|u^{(m)}(t)\right|^{2} d t \\
=(-1)^{n-2 m} \sum_{i=1}^{m} \int_{t_{0}}^{t_{1}}(t-a)^{n-2 m} p_{i}(t) u^{(i-1)}(t) u(t) d t \\
+(-1)^{n-2 m} \int_{t_{0}}^{t_{1}}(t-a)^{n-2 m} q(t) u(t) d t .
\end{aligned}
$$

According to Lemmas 2.7, 2.6', and conditions (1.20), we have

$$
\begin{aligned}
& (-1)^{n-m} \int_{t_{0}}^{a_{0}}(t-a)^{n-2 m} p_{1}(t) u^{2}(t) u(t) d t \leq \int_{t_{0}}^{a_{0}}(t-a)^{n-2 m}\left[(-1)^{n-m} p_{1}(t)\right]_{+} u^{2}(t) d t \\
& \leq \frac{(2 m-1) 2^{2 m}}{[(2 m-1) ! !]^{2}} \ell_{11} \int_{t_{0}}^{a_{0}}\left|u^{(m)}(t)\right|^{2} d t \\
& \left|\int_{t_{0}}^{a_{0}}(t-a)^{n-2 m} p_{i}(t) u^{(i-1)}(t) u(t) d t\right| \\
& \leq \frac{(2 m-i) 2^{2 m-i+1}}{(2 m-1) ! !(2 m-2 i+1) ! !} \ell_{1 i} \int_{t_{0}}^{a_{0}}\left|u^{(m)}(t)\right|^{2} d t \quad(i=2, \ldots, m), \\
& (-1)^{n-m} \int_{b_{0}}^{t_{1}}(t-a)^{n-2 m} p_{1}(t) u^{2}(t) d t \leq \int_{b_{0}}^{t_{1}}(t-a)^{n-2 m}\left[(-1)^{n-m} p_{1}(t)\right]_{+} u^{2}(t) d t \\
& \leq \frac{(2 m-1) 2^{2 m}}{[(2 m-1) ! !]^{2}} \ell_{21} \int_{b_{0}}^{t_{1}}\left|u^{(m)}(t)\right|^{2} d t \text {, } \\
& \left|\int_{b_{0}}^{t_{1}}(t-a)^{n-2 m} p_{i}(t) u^{(i-1)}(t) u(t) d t\right| \\
& \leq \frac{(2 m-i) 2^{2 m-i+1}}{(2 m-1) ! !(2 m-2 i+1) ! !} \ell_{2 i} \int_{b_{0}}^{t_{1}}\left|u^{(m)}(t)\right|^{2} d t \quad(i=2, \ldots, m) .
\end{aligned}
$$

If along with this we take into account inequalities (2.93), we find

$$
\begin{aligned}
(-1)^{n-2 m} \sum_{i=1}^{m} \int_{t_{0}}^{t_{1}}(t-a)^{n-2 m} p_{i}(t) u^{(i-1)}(t) u(t) d t \\
\leq\left|\sum_{i=1}^{m} \int_{a_{0}}^{b_{0}}(t-a)^{n-2 m} p_{i}(t) u^{(i-1)}(t) u(t) d t\right| \\
\quad+\left(\mu_{n}-\gamma\right)\left(\int_{t_{0}}^{a_{0}}\left|u^{(m)}(t)\right|^{2} d t+\int_{b_{0}}^{t_{1}}\left|u^{(m)}(t)\right|^{2} d t\right) \\
\leq\left|\sum_{i=1}^{m} \int_{a_{0}}^{b_{0}}(t-a)^{n-2 m} p_{i}(t) u^{(i-1)}(t) u(t) d t\right|+\left(\mu_{n}-\gamma\right) \int_{t_{0}}^{t_{1}}\left|u^{(m)}(t)\right|^{2} d t .
\end{aligned}
$$


On the other hand, if we put $c=(a+b) / 2$, then again on the basis of Lemmas 2.7 and $2.6^{\prime}$ we get

$$
\begin{aligned}
& \left|\int_{t_{0}}^{t_{1}}(t-a)^{n-2 m} q(t) u(t) d t\right| \\
& \leq\left|\int_{t_{0}}^{c}(t-a)^{n-2 m} q(t) u(t) d t\right|+\left|\int_{c}^{t_{1}}(t-a)^{n-2 m} q(t) u(t) d t\right| \\
& =\left|\int_{t_{0}}^{c}\left[(n-2 m) u(t)+(t-a)^{n-2 m} u^{\prime}(t)\right]\left(\int_{t}^{c} q(s) d s\right) d t\right| \\
& +\left|\int_{c}^{t_{1}}\left[(n-2 m) u(t)+(t-a)^{n-2 m} u^{\prime}(t)\right]\left(\int_{c}^{t} q(s) d s\right) d t\right| \\
& \leq\left[(n-2 m)\left(\int_{t_{0}}^{c} \frac{u^{2}(t) d t}{(t-a)^{2 m}}\right)^{1 / 2}+\left(\int_{t_{0}}^{c} \frac{u^{\prime 2}(t) d t}{(t-a)^{2 m-2}}\right)^{1 / 2}\right] \\
& \times\left(\int_{t_{0}}^{c}(t-a)^{2 n-2 m-2}\left(\int_{t}^{c} q(s) d s\right)^{2} d t\right)^{1 / 2} \\
& +(b-a)\left[(n-2 m)\left(\int_{c}^{t_{1}} \frac{u^{2}(t) d t}{(b-t)^{2 m}}\right)^{1 / 2}+\left(\int_{c}^{t_{1}} \frac{u^{\prime 2}(t) d t}{(b-t)^{2 m-2}}\right)^{1 / 2}\right] \\
& \times\left(\int_{c}^{t_{1}}(b-t)^{2 m-2}\left(\int_{c}^{t} q(s) d s\right)^{2} d t\right)^{1 / 2} \\
& \leq 2^{m+1}(1+b-a)\left[\left(\int_{t_{0}}^{c}\left|u^{(m)}(t)\right|^{2} d t\right)^{1 / 2}+\left(\int_{c}^{t_{1}}\left|u^{(m)}(t)\right|^{2} d t\right)^{1 / 2}\right]\|q\|_{\tilde{L}_{2 n-2 m-2,2 m-2}^{2}} \\
& \leq \frac{\gamma}{2} \int_{t_{0}}^{t_{1}}\left|u^{(m)}(t)\right|^{2} d t+2^{2 m+1}(1+b-a)^{2} \gamma^{-1}\|q\|_{\widetilde{L}_{2 n-2 m-2,2 m-2}^{2}}^{2} .
\end{aligned}
$$

In view of inequalities (2.97), (2.98) and notation (2.94), equality (2.95) results in estimate (2.90).

The proof of the following lemma is analogous to that of Lemma 2.11.

LEMma 2.13. Let conditions (1.12), (1.24), and (1.25) hold, where $h_{i}(i=1, \ldots, m)$ are functions given by equalities (1.13), $\left.a_{0} \in\right] a, b\left[\right.$, and $\ell_{i}(i=1, \ldots, m)$ are nonnegative numbers. Then there exists a positive constant $r_{0}$ such that for any $\left.t_{0} \in\right] a, a_{0}\left[\right.$ and $\left.\left.q \in \tilde{L}_{2 n-2 m-2}^{2}(] a, b\right]\right)$, an arbitrary solution $\left.\left.u \in C_{\mathrm{loc}}^{n-1}(] a, b\right]\right)$ of $(1.1)$, satisfying the conditions

$$
u^{(i-1)}\left(t_{0}\right)=0 \quad(i=1, \ldots, m), \quad u^{(j-1)}(b)=0 \quad(j=m+1, \ldots, n),
$$

also satisfies the condition

$$
\int_{t_{0}}^{b}\left|u^{(m)}(t)\right|^{2} d t \leq r_{0}\left(\left|\sum_{i=1}^{m} \int_{a_{0}}^{b}(t-a)^{n-2 m} p_{i}(t) u^{(i-1)}(t) u(t) d t\right|+\|q\|_{\widetilde{L}_{2 n-2 m-2}^{2}}^{2}\right) .
$$


Lemma 2.14. Let conditions (1.10), (1.20), and (1.21) hold, and in the case, where $n$ is odd, in addition condition (1.11) be fulfilled, where $h_{i}(i=1, \ldots, m)$ are functions given by equalities (1.13), $\left.a_{0} \in\right] a, b\left[, b_{0} \in\right] a_{0}, b\left[\right.$, and $\ell_{1 i}, \ell_{2 i}(i=1, \ldots, m)$ are nonnegative numbers. Let, moreover, the homogeneous problem (1.1 $),(1.2)$ in the space $\widetilde{C}^{n-1, m}(] a, b[)$ have only a trivial solution. Then there exist $\delta \in] 0,(b-a) / 2\left[\right.$ and $r>0$ such that for any $\left.t_{0} \in\right] a, a+\delta$ ], $t_{1} \in\left[b-\delta, b\left[\right.\right.$, and $q \in \widetilde{L}_{2 n-2 m-2,2 m-2}^{2}(] a, b[)$ problem (1.1), (2.89) is uniquely solvable in the space $\widetilde{C}_{\mathrm{loc}}^{n-1}(] a, b[)$ and its solution admits the estimate

$$
\left(\int_{t_{0}}^{t_{1}}\left|u^{(m)}(t)\right|^{2} d t\right)^{1 / 2} \leq r\|q\|_{\tilde{L}_{2 n-2 m-2,2 m-2}^{2}}
$$

Proof. First note that for arbitrarily fixed $\left.t_{0} \in\right] a, a+\delta\left[, t_{1} \in\right] b-\delta, b\left[\right.$, and $q \in L\left(\left[t_{0}, t_{1}\right]\right)$ problem $(1.1),(2.89)$ is regular and has the Fredholm property in the space $\widetilde{C}^{n-1}\left(\left[t_{0}, t_{1}\right]\right)$.

Assume now that the lemma is not true. Then by virtue of the above-analysis, for an arbitrary natural $k$ there exist

$$
\left.t_{0 k} \in\right] a, a+\frac{b-a}{2 k}\left[, \quad t_{1 k} \in\right] b-\frac{b-a}{2 k}, b[
$$

and a function $q_{k} \in \tilde{L}_{2 n-2 m-2,2 m-2}^{2}(] a, b[)$ such that problem (2.44), (2.45) has a solution $u_{k} \in \widetilde{C}_{\text {loc }}^{n-1}(] a, b[)$ satisfying the inequality

$$
\left(\int_{t_{0 k}}^{t_{1 k}}\left|u_{k}^{(m)}(t)\right|^{2} d t\right)^{1 / 2}>k\left\|q_{k}\right\|_{\tilde{L}_{2 n-2 m-2,2 m-2}^{2}}
$$

Suppose

$$
v_{k}(t)=\left(\int_{t_{0 k}}^{t_{1 k}}\left|u_{k}^{(m)}(t)\right|^{2} d t\right)^{-1 / 2} u_{k}(t), \quad q_{0 k}(t)=\left(\int_{t_{0 k}}^{t_{1 k}}\left|u_{k}^{(m)}(t)\right|^{2} d t\right)^{-1 / 2} q_{k}(t)
$$

Then $v_{k}$ is a solution of the problem

$$
\begin{gathered}
v^{(n)}=\sum_{i=1}^{m} p_{i}(t) v^{(i-1)}+q_{0 k}(t), \\
v^{(i-1)}\left(t_{0 k}\right)=0 \quad(i=1, \ldots, m), \quad v^{(i-1)}\left(t_{1 k}\right)=0 \quad(i=1, \ldots, n-m) .
\end{gathered}
$$

Moreover,

$$
\int_{t_{0 k}}^{t_{1 k}}\left|v_{k}^{(m)}(t)\right|^{2} d t=1, \quad\left\|q_{0 k}\right\|_{\widetilde{L}_{2 n-2 m-2,2 m-2}^{2}}<\frac{1}{k} \quad(k=1,2, \ldots) .
$$


On the other hand, by Lemmas 2.9 and 2.11, we have

$$
\begin{gathered}
\left.\lim _{k \rightarrow+\infty} v_{k}^{(i-1)}(t)=0 \text { uniformly in }\right] a, b[\quad(i=1, \ldots, n), \\
1 \leq r_{0}\left(\left|\sum_{i=1}^{m} \int_{a_{0}}^{b_{0}}(t-a)^{n-2 m} p_{i}(t) v_{k}^{(i-1)}(t) v_{k}(t) d t\right|+k^{-2}\right) \quad(k=1,2, \ldots),
\end{gathered}
$$

where $r_{0}$ is a positive constant independent of $k$. Thus if we pass to the limit in the last inequality as $k \rightarrow+\infty$, then we obtain the contradiction $1 \leq 0$, which proves the lemma.

Analogously we can prove the following lemma if we apply Lemmas 2.10 and 2.13 instead of Lemmas 2.9 and 2.11.

Lemma 2.15. Let conditions (1.12), (1.24), and (1.25) hold, where $h_{i}(i=1, \ldots, m)$ are functions given by equalities (1.13), $\left.a_{0} \in\right] a, b\left[\right.$, and $\ell_{i}(i=1, \ldots, m)$ are nonnegative numbers. Let, moreover, the homogeneous problem (1.1 $),(1.3)$ in the space $\left.\left.\widetilde{C}^{n-1, m}(] a, b\right]\right)$ have only a trivial solution. Then there exist $\delta \in] 0, b-a\left[\right.$ and $r>0$ such that for any $\left.\left.t_{0} \in\right] a, a+\delta\right]$ and $\left.\left.q \in \widetilde{L}_{2 n-2 m-2}^{2}(] a, b\right]\right)$ problem (1.1), (2.99) is uniquely solvable in the space $\left.\left.\widetilde{C}_{\mathrm{loc}}^{n-1}(] a, b\right]\right)$ and its solution admits the estimate

$$
\left(\int_{t_{0}}^{b}\left|u^{(m)}(t)\right|^{2} d t\right)^{1 / 2} \leq r\|q\|_{\tilde{L}_{2 n-2 m-2}^{2}}
$$

\section{Proof of the main results}

Proof of Theorem 1.3 (Theorem 1.5). Suppose problem (1.1 $\left.1_{0}\right),(1.2)$ (problem $\left(1.1_{0}\right)$, (1.3)) has only a trivial solution, and $r$ and $\delta$ are the numbers appearing in Lemma 2.14 (in Lemma 2.15). Set

$$
t_{0 k}=a+\frac{\delta}{k}, \quad t_{1 k}=b-\frac{\delta}{k} \quad(k=1,2, \ldots)
$$

By Lemma 2.14 (Lemma 2.15) for every natural $k$ problem (1.1), (2.45) (problem (1.1), $(2.46))$ in the space $\widetilde{C}_{\mathrm{loc}}^{n-1}(] a, b[)$ (in the space $\left.\left.\left.\widetilde{C}_{\mathrm{loc}}^{n-1}(] a, b\right]\right)\right)$ has a unique solution $u_{k}$ and

$$
\left(\int_{t_{0 k}}^{t_{1 k}}\left|u_{k}^{(m)}(t)\right|^{2} d t\right)^{1 / 2} \leq r\|q\|_{\tilde{L}_{2 n-2 m-2,2 m-2}^{2}} \quad\left(\left(\int_{t_{0 k}}^{b}\left|u_{k}^{(m)}(t)\right|^{2} d t\right)^{1 / 2} \leq r\|q\|_{\tilde{L}_{2 n-2 m-2}^{2}}\right)
$$

Hence by Lemma 2.9 (by Lemma 2.10) it follows that problem (1.1), (1.2) (problem (1.1), (1.3)) in the space $\widetilde{C}^{n-1, m}(] a, b[)$ (in the space $\left.\left.\left.\widetilde{C}^{n-1, m}(] a, b\right]\right)\right)$ is uniquely solvable and its solution admits estimate (1.15). Therefore problem (1.1), (1.2) (problem (1.1), (1.3)) has the Fredholm property since the constant $r$ does not depend on $q$. 
Proof of Corollary 1.4. By conditions (1.23), there exist positive constants $\ell_{1 i}, \ell_{2 i}(i=1, \ldots$, $m)$, satisfying inequalities (1.21), such that

$$
\lambda_{1 i}<(2 m-i) \ell_{1 i}, \quad \lambda_{2 i}<(2 m-i) \ell_{2 i} \quad(i=1, \ldots, m) .
$$

Choose $\left.a_{0} \in\right] a, b\left[\right.$ and $\left.b_{0} \in\right] a_{0}, b[$ so that

$$
\begin{aligned}
& \frac{\lambda_{1 i}}{2 m-i}+\lambda_{2 i} \int_{a}^{a_{0}} \frac{(s-a)^{2 m-i} d s}{(b-s)^{2 m-i+1}}+\int_{a}^{a_{0}}(s-a)^{n-i} p_{0 i}(s) d s<\ell_{1 i} \quad(i=1, \ldots, m), \\
& \frac{\lambda_{2 i}}{2 m-i}+\lambda_{1 i} \int_{b_{0}}^{b} \frac{(b-s)^{2 m-i} d s}{(s-a)^{n-i+1}}+\int_{b_{0}}^{b}(b-s)^{2 m-i} p_{0 i}(s) d s<\ell_{2 i} \quad(i=1, \ldots, m) .
\end{aligned}
$$

Then, according to (1.13), inequalities (1.22) yield inequalities (1.20). Therefore all the conditions of Theorem 1.3 are fulfilled which guarantee the validity of Corollary 1.4.

Analogously, Corollary 1.6 follows from Theorem 1.5 since conditions (1.26) and (1.27) guarantee conditions (1.24) and (1.25) for some $\left.a_{0} \in\right] a, b\left[\right.$ and $\ell_{i}>0(i=1, \ldots, m)$.

Proof of Theorem 1.7. It is sufficient to show that if $u \in \widetilde{C}_{\mathrm{loc}}^{n-1}(] a, b[)$ is a solution of problem $\left(1.1_{0}\right),(1.2)$ (problem $\left.\left(1.1_{0}\right),(1.3)\right)$, then

$$
\int_{a}^{b}\left|u^{(m)}(t)\right|^{2} d t<+\infty
$$

For an arbitrary $\left.t_{0} \in\right] a, b[$ we have

$$
\begin{aligned}
u^{(m)}(t)= & \sum_{j=m+1}^{n} \frac{\left(t-t_{0}\right)^{j-m-1}}{(j-m-1) !} u^{(j-1)}\left(t_{0}\right) \\
& +\frac{1}{(n-m-1) !} \int_{t_{0}}^{t}(t-s)^{n-m-1}\left(\sum_{i=1}^{m} p_{i}(s) u^{(i-1)}(s)\right) d s
\end{aligned}
$$

Hence, according to conditions (1.2) and (1.28) (conditions (1.3) and (1.28)), it is obvious that $u^{(m)} \in L([a, b])$. Put

$$
\begin{gathered}
p(t)=\sum_{i=1}^{m}(t-a)^{n-i}\left|p_{i}(t)\right| \\
v(t)=\int_{a}^{t}\left|u^{(m)}(s)\right| d s, \quad w\left(t_{0}\right)=\sum_{j=m+1}^{n} \frac{\left(t_{0}-a\right)^{j-m-1}}{(j-m-1) !}\left|u^{(j-1)}\left(t_{0}\right)\right|,
\end{gathered}
$$

and choose $\left.t_{0} \in\right] a, b[$ such that

$$
\int_{a}^{t_{0}} p(s) d s<\frac{1}{2} .
$$


Then in view of (1.2), ((1.3)), and (3.5) we find

$$
\begin{aligned}
\left|u^{(i-1)}(t)\right|= & \frac{1}{(m-i) !}\left|\int_{a}^{t}(t-s)^{m-i} u^{(m)}(s) d s\right| \leq(t-a)^{m-i} v(t) \quad(i=1, \ldots, m), \\
& \left|u^{(m)}(t)\right| \leq w\left(t_{0}\right)+\int_{t}^{t_{0}} \frac{p(s) v(s)}{s-a} d s \quad \text { for } a<t \leq t_{0}, \\
v(t) \leq & w\left(t_{0}\right)(t-a)+\int_{a}^{t}\left(\int_{\tau}^{t_{0}} \frac{p(s) v(s)}{s-a} d s\right) d \tau \\
= & w\left(t_{0}\right)(t-a)+(t-a) \int_{t}^{t_{0}} \frac{p(s) v(s)}{s-a} d s+\int_{a}^{t} p(s) v(s) d s \\
\leq & w\left(t_{0}\right)(t-a)+(t-a) \int_{t}^{t_{0}} \frac{p(s) v(s)}{s-a} d s+\frac{1}{2} v(t) \quad \text { for } a<t<t_{0},
\end{aligned}
$$

and, consequently,

$$
\frac{v(t)}{t-a} \leq w\left(t_{0}\right)+2 \int_{t}^{t_{0}} p(s) \frac{v(s)}{s-a} d s \quad \text { for } a<t<t_{0} .
$$

The last inequality, by the Gronwall-Bellman lemma, results in

$$
\frac{v(t)}{t-a} \leq w\left(t_{0}\right) \exp \left(2 \int_{t}^{t_{0}} p(s) d s\right) \leq w\left(t_{0}\right) \exp (1) \quad \text { for } a<t \leq t_{0} .
$$

Due to this inequality, from (3.9) we get

$$
\left|u^{(m)}(t)\right| \leq(1+\exp (1)) w\left(t_{0}\right) \quad \text { for } a<t \leq t_{0} .
$$

Analogously we can show that $u^{(m)}$ is bounded in the neighborhood of the point $b$. Therefore condition (3.5) is satisfied.

Proof of Theorem 1.9. By Theorem 1.3, from inequalities (1.21) and (1.49) it follows that problem (1.1), (1.2) has the Fredholm property. Thus to prove Theorem 1.9, it suffices to show that the homogeneous problem $\left(1.1_{0}\right),(1.2)$ in the space $\widetilde{C}^{n-1, m}(] a, b[)$ has only a trivial solution.

Suppose $u \in \widetilde{C}^{n-1, m}(] a, b[)$ is a solution of problem (1.1 $)$, (1.2). Put

$$
\rho_{1}(t)=\int_{a}^{t}\left|u^{(m)}(\tau)\right|^{2} d \tau, \quad \rho_{2}(t)=\int_{t}^{b}\left|u^{(m)}(\tau)\right|^{2} d \tau, \quad \rho=\int_{a}^{b}\left|u^{(m)}(\tau)\right|^{2} d \tau .
$$

Multiplying $\left(1.1_{0}\right)$ by $(-1)^{n-m}(t-a)^{n-2 m} u(t)$ and then integrating from $s$ to $t$, by Lemma 2.12 we obtain

$$
\begin{aligned}
w_{n}(t) & -w_{n}(s)+\mu_{n} \int_{s}^{t}\left|u^{(m)}(\tau)\right|^{2} d \tau \\
& =(-1)^{n-m} \sum_{i=1}^{m} \int_{s}^{t}(\tau-a)^{n-2 m} p_{i}(\tau) u^{(i-1)}(\tau) u(\tau) d \tau \quad \text { for } a<s \leq t<b,
\end{aligned}
$$


where $\mu_{n}$ and $w_{n}$ are the number and the function, respectively, given by equalities (2.92). Moreover, it follows from Lemma 2.8,

$$
\liminf _{s \rightarrow a}\left|w_{n}(s)\right|=0, \quad \liminf _{t \rightarrow b}\left|w_{n}(t)\right|=0 .
$$

By virtue of Lemmas 2.7, 2.6', and conditions (1.49), we have

$$
\begin{aligned}
(-1)^{n-m} & \int_{s}^{t}(\tau-a)^{n-2 m} p_{i}(\tau) u^{(i-1)}(\tau) u(\tau) d \tau \\
\leq & {\left[\rho_{1}(s)+\frac{(2 m-i) 2^{2 m-i+1}}{(2 m-1) ! !(2 m-2 i+1) ! !} \rho_{1}\left(t_{0}\right)\right] \ell_{1 i} } \\
& +\left[\rho_{2}(t)+\frac{(2 m-i) 2^{2 m-i+1}}{(2 m-1) ! !(2 m-2 i+1) ! !} \rho_{2}\left(t_{0}\right)\right] \ell_{2 i} \quad \text { for } a<s \leq t_{0} \leq t<b(i=1, \ldots, m) .
\end{aligned}
$$

Due to (1.21), the number $\gamma \in] 0,1[$ can be chosen so that inequalities $(2.93)$ would be satisfied.

According to (2.93) and (3.16), (3.14) implies

$$
\begin{aligned}
w_{n}(t) & -w_{n}(s)+\mu_{n} \int_{s}^{t}\left|u^{(m)}(\tau)\right|^{2} d \tau \\
& \leq\left(\sum_{i=1}^{m} \ell_{1 i}\right) \rho_{1}(s)+\left(\sum_{i=1}^{m} \ell_{2 i}\right) \rho_{2}(t)+\left(\mu_{n}-\gamma\right)\left(\rho_{1}\left(t_{0}\right)+\rho_{2}\left(t_{0}\right)\right) \\
& =\left(\sum_{i=1}^{m} \ell_{1 i}\right) \rho_{1}(s)+\left(\sum_{i=1}^{m} \ell_{2 i}\right) \rho_{2}(t)+\left(\mu_{n}-\gamma\right) \rho .
\end{aligned}
$$

Hence, by equalities (3.15), we find

$$
\mu_{n} \rho \leq\left(\mu_{n}-\gamma\right) \rho
$$

and consequently, $\rho=0$. However,

$$
|u(t)| \leq \frac{\rho}{(m-1) !}(t-a)^{m-1 / 2} \text { for } a<t<b,
$$

and therefore, $u(t) \equiv 0$.

The proof of Theorem 1.11 is analogous to that of Theorem 1.9. The only difference is that instead of Theorem 1.3, inequalities (1.21) and (1.49) Theorem 1.5, inequalities (1.25) and (1.52) are applied.

To convince ourselves of the validity of Corollary 1.10 (Corollary 1.12), it suffices to note that inequalities (1.23), (1.50), and (1.51) (inequalities (1.27) and (1.53)) guarantee inequalities (1.21), (1.49) (inequalities (1.25), (1.52)), where

$$
\ell_{1 i}=\frac{\lambda_{1 i}}{2 m-i}, \quad \ell_{2 i}=\frac{\lambda_{2 i}}{2 m-i} \quad\left(\ell_{i}=\frac{\lambda_{i}}{2 m-i}\right) \quad(i=1, \ldots, m) .
$$


Remark 3.1. From Lemmas 2.3 and 2.4 it follows that if either condition (1.16) or condition (1.17) is fulfilled, then condition (1.18) holds as well, and the inequalities

$$
\begin{array}{lr}
\|q\|_{\tilde{L}_{2 n-2 m-2,2 m-2}^{2}} \leq \gamma\|q\|_{L_{2 n-2 m, 2 m}^{2}} & \left(\|q\|_{\widetilde{L}_{2 n-2 m-2}^{2}} \leq \gamma\|q\|_{L_{2 n-2 m, 0}^{2}}\right), \\
\|q\|_{\tilde{L}_{2 n-2 m-2,2 m-2}^{2}} \leq \gamma\|q\|_{L_{n-m-1 / 2, m-1 / 2}} & \left(\|q\|_{\tilde{L}_{2 n-2 m-2}^{2}} \leq \gamma\|q\|_{L_{n-m-1 / 2,0}}\right)
\end{array}
$$

are valid, respectively, where $\gamma$ is a positive constant independent of $q$. Thus in those cases estimate (1.15) yields estimates (1.19), where $r_{0}=\gamma r$. Therefore Remark 1.2 is valid.

\section{Acknowledgment}

This work was supported by GRDF Grant no. 3318.

\section{References}

[1] R. P. Agarwal, Focal boundary value problems for differential and difference equations, Mathematics and Its Applications, vol. 436, Kluwer Academic Publishers, Dordrecht, 1998.

[2] R. P. Agarwal and D. O’Regan, Singular differential and integral equations with applications, Kluwer Academic Publishers, Dordrecht, 2003.

[3] I. T. Kiguradze, On a singular boundary value problem, Journal of Mathematical Analysis and Applications. 30 (1970), no. 3, 475-489.

[4] _ On a singular multi-point boundary value problem, Annali di Matematica Pura ed Applicata. Series IV. 86 (1970), 367-399.

[5] I. Kiguradze, Some singular boundary value problems for ordinary differential equations, Tbilisi University Press, Tbilisi, 1975.

[6] __ Some optimal conditions for the solvability of two-point singular boundary value problems, Functional Differential Equations 10 (2003), no. 1-2, 259-281, Functional differential equations and applications (Beer-Sheva, 2002).

[7] _ On two-point boundary value problems for higher order singular ordinary differential equations, Georgian Academy of Sciences. A. Razmadze Mathematical Institute. Memoirs on Differential Equations and Mathematical Physics 31 (2004), 101-107.

[8] I. T. Kiguradze and T. A. Chanturia, Asymptotic properties of solutions of nonautonomous ordinary differential equations, Mathematics and Its Applications (Soviet Series), vol. 89, Kluwer Academic Publishers, Dordrecht, 1993, Translated from the 1985 Russian original.

[9] I. Kiguradze and B. Půža, Conti-Opial type existence and uniqueness theorems for nonlinear singular boundary value problems, Functional Differential Equations 9 (2002), no. 3-4, 405-422, Dedicated to L. F. Rakhmatullina and N. V. Azbelev on the occasion of their seventieth and eightieth birthdays.

[10] I. Kiguradze, B. Půža, and I. P. Stavroulakis, On singular boundary value problems for functional differential equations of higher order, Georgian Mathematical Journal 8 (2001), no. 4, 791-814.

[11] I. T. Kiguradze and B. L. Shekhter, Singular boundary value problems for second-order ordinary differential equations, Current problems in mathematics. Newest results, Vol. 30 (Russian), Itogi Nauki i Tekhniki, Akad. Nauk SSSR Vsesoyuz. Inst. Nauchn. i Tekhn. Inform., Moscow, 1987, pp. 105-201, 204, Translated in J. Soviet Math. 43 (1988), no. 2, 2340-2417.

[12] I. Kiguradze and G. Tskhovrebadze, On two-point boundary value problems for systems of higherorder ordinary differential equations with singularities, Georgian Mathematical Journal 1 (1994), no. $1,31-45$. 
[13] V. E. Maĭorov, On the existence of solutions of higher-order singular differential equations, Rossǐ̌skaya Akademiya Nauk. Matematicheskie Zametki 51 (1992), no. 3, 75-84 (Russian), translation in Math. Notes 51 (1992), no. 3-4, 274-281.

[14] B. Půža, On a singular two-point boundary value problem for the nonlinear mth-order differential equation with deviating arguments, Georgian Mathematical Journal 4 (1997), no. 6, 557-566.

[15] B. Půža and A. Rabbimov, On a weighted boundary value problem for a system of singular functional-differential equations, Georgian Academy of Sciences. A. Razmadze Mathematical Institute. Memoirs on Differential Equations and Mathematical Physics 21 (2000), 125-130.

[16] G. D. Tskhovrebadze, On a multipoint boundary value problem for linear ordinary differential equations with singularities, Universitatis Masarykianae Brunensis. Facultas Scientiarum Naturalium. Archivum Mathematicum 30 (1994), no. 3, 171-206.

[17] G. Tskhovrebadze, On the modified boundary value problem of de la Vallée-Poussin for nonlinear ordinary differential equations, Georgian Mathematical Journal 1 (1994), no. 4, 429-458.

[18] P. J. Y. Wong and R. P. Agarwal, Singular differential equations with $(n, p)$ boundary conditions, Mathematical and Computer Modelling 28 (1998), no. 1, 37-44.

R. P. Agarwal: Department of Mathematical Sciences, Florida Institute of Technology,

Melbourne, FL 32901, USA

E-mail address: agarwal@fit.edu

I. Kiguradze: A. Razmadze Mathematical Institute, Georgian Academy of Sciences,

1 M. Aleksidze Street, Tbilisi 0193, Georgia

E-mail address: kig@rmi.acnet.ge 